EUROPEAN ORGANIZATION FOR NUCLEAR RESEARCH

CERN-PPE/97-107

8 August 1997

\title{
Search for charginos, neutralinos and gravitinos at LEP
}

\author{
DELPHI Collaboration
}

\begin{abstract}
An update of the searches for charginos and neutralinos in DELPHI is presented, based mainly on recent data collected at centre-of-mass energies of $161 \mathrm{GeV}$ and $172 \mathrm{GeV}$. No signal is found. For a sneutrino with mass above $300 \mathrm{GeV} / c^{2}$ and a mass difference between the chargino and the lightest neutralino above $10 \mathrm{GeV} / c^{2}$, the lower limit at $95 \%$ confidence level on the chargino mass ranges from $84.3 \mathrm{GeV} / c^{2}$ to the kinematical limit $\left(86.0 \mathrm{GeV} / c^{2}\right)$, depending on the mixing parameters. The limit decreases for lower chargino-neutralino mass differences. The limit in the case of a light sneutrino is $67.6 \mathrm{GeV} / c^{2}$, provided that that there is no light sneutrino with a mass within $10 \mathrm{GeV} / c^{2}$ below the chargino mass. Upper limits on neutralino pair production cross-sections of about a picobarn are derived. The $\left(\mu, M_{2}\right)$ domain excluded in the MSSMGUT scenario is determined by combining the neutralino and chargino searches. These results imply a limit on the mass of the lightest neutralino which, for a heavy sneutrino, is constrained to be above $24.9 \mathrm{GeV} / c^{2}$ for $\tan \beta \geq 1$. The search has also been extended to the case where the lightest neutralino is unstable and decays into a photon and a gravitino.
\end{abstract}

(To be submitted to Zeit. f. Physik C) 
P.Abreu $^{21}$, W.Adam ${ }^{49}$, T.Adye ${ }^{36}$, I.Ajinenko ${ }^{41}$, G.D.Alekseev ${ }^{16}$, R.Alemany ${ }^{48}$, P.P.Allport ${ }^{22}$, S.Almehed ${ }^{24}$, U.Amaldi $^{9}$, S.Amato ${ }^{46}$, P.Andersson ${ }^{43}$, A.Andreazza ${ }^{9}$, P.Antilogus ${ }^{9}$, W-D.Apel ${ }^{17}$, Y.Arnoud ${ }^{14}$, B.Asman $^{43}$, J-E.Augustin ${ }^{25}$, A.Augustinus ${ }^{30}$, P.Baillon ${ }^{9}$, P.Bambade ${ }^{19}$, M.Barbi ${ }^{46}$, D.Y.Bardin ${ }^{16}, \quad$ G.Barker ${ }^{9}$, A.Baroncelli $^{39}$, O.Barring ${ }^{24}$, M.J.Bates ${ }^{36}$, M.Battaglia $^{15}$, M.Baubillier ${ }^{23}$, J.Baudot ${ }^{38}$, K-H.Becks ${ }^{51}$, M.Begalli ${ }^{6}$, P.Beilliere $^{8}$, Yu.Belokopytov ${ }^{9,52}$, K.Belous ${ }^{41}$, A.C.Benvenuti ${ }^{5}$, C.Berat ${ }^{14}$, M.Berggren ${ }^{46}$, D.Bertini $^{25}$, D.Bertrand ${ }^{2}$, M.Besancon ${ }^{38}$, F.Bianchi $^{44}$, M.Bigi $^{44}$, M.S.Bilenky ${ }^{16}$, P.Billoir ${ }^{23}$, M-A.Bizouard ${ }^{19}$, D.Bloch $^{10}$, M.Blume $^{51}$, M.Bonesini ${ }^{27}$, W.Bonivento ${ }^{27}$, M.Boonekamp ${ }^{38}$, P.S.L.Booth ${ }^{22}$, A.W.Borgland ${ }^{4}$, G.Borisov ${ }^{38,41}$, C.Bosio $^{39}$, O.Botner ${ }^{47}$, E.Boudinov ${ }^{30}$, B.Bouquet ${ }^{19}$, C.Bourdarios ${ }^{19}$, T.J.V.Bowcock ${ }^{22}$, M.Bozzo ${ }^{13}$, P.Branchini $^{39}$, K.D.Brand ${ }^{35}$, T.Brenke ${ }^{51}$, R.A.Brenner ${ }^{47}$, R.C.A.Brown ${ }^{9}$, P.Bruckman ${ }^{18}$, J-M.Brunet ${ }^{8}$, L.Bugge $^{32}$, T.Buran ${ }^{32}$, T.Burgsmueller ${ }^{51}$, P.Buschmann ${ }^{51}$, S.Cabrera ${ }^{48}$, M.Caccia ${ }^{27}$, M.Calvi ${ }^{27}$, A.J.Camacho $\operatorname{Rozas}^{40}$, T.Camporesi ${ }^{9}$, V.Canale ${ }^{37}, \quad$ M.Canepa ${ }^{13}$, F.Carena ${ }^{9}$, L.Carroll ${ }^{22}$, C.Caso $^{13}$, M.V.Castillo Gimenez ${ }^{48}$, A.Cattai ${ }^{9}$, F.R.Cavallo ${ }^{5}$, V.Chabaud ${ }^{9}$, M.Chapkin ${ }^{41}$, Ph.Charpentier ${ }^{9}$, L.Chaussard $^{25}$, P.Checchia $^{35}$, G.A.Chelkov ${ }^{16}$, M.Chen ${ }^{2}$, R.Chierici ${ }^{44}$, P.Chliapnikov ${ }^{41}$, P.Chochula ${ }^{7}$, V.Chorowicz ${ }^{25}$, J.Chudoba $^{29}$, V.Cindro ${ }^{42}$, P.Collins ${ }^{9}$, M.Colomer ${ }^{48}$, R.Contri ${ }^{13}$, E.Cortina ${ }^{48}$, G.Cosme ${ }^{19}$, F.Cossutti ${ }^{45}$, J-H.Cowell ${ }^{22}$, H.B.Crawley ${ }^{1}$, D.Crennell ${ }^{36}$, G.Crosetti ${ }^{13}$, J.Cuevas Maestro ${ }^{33}$, S.Czellar ${ }^{15}$, J.Dahm ${ }^{51}$, B.Dalmagne $^{19}$, G.Damgaard ${ }^{28}$, P.D.Dauncey ${ }^{36}$, M.Davenport ${ }^{9}$, W.Da Silva ${ }^{23}$, A.Deghorain ${ }^{2}$, G.Della Ricca ${ }^{45}$, P.Delpierre $^{26}$, N.Demaria ${ }^{34}$, A.De Angelis ${ }^{9}$, W.De Boer ${ }^{17}$, S.De Brabandere ${ }^{2}$ C.De Clercq ${ }^{2}$, C.De La Vaissiere ${ }^{23}$, B.De Lotto ${ }^{45}$, A.De Min ${ }^{35}$, L.De Paula ${ }^{46}$, H.Dijkstra ${ }^{9}$, L.Di Ciaccio ${ }^{37}$, A.Di Diodato ${ }^{37}$, A.Djannati $^{8}$, J.Dolbeau ${ }^{8}$, K.Doroba ${ }^{50}$, M.Dracos ${ }^{10}$, J.Drees ${ }^{51}$, K.-A.Drees ${ }^{51}$, M.Dris ${ }^{31}$, J-D.Durand ${ }^{25,9}$, D.Edsall $^{1}$, R.Ehret ${ }^{17}$, G.Eigen ${ }^{4}$, T.Ekelof ${ }^{47}$, G.Ekspong ${ }^{43}$, M.Elsing ${ }^{9}$, J-P.Engel ${ }^{10}$, B.Erzen ${ }^{42}$, M.Espirito Santo ${ }^{21}$, E.Falk ${ }^{24}$, G.Fanourakis ${ }^{11}$, D.Fassouliotis ${ }^{45}$, M.Feindt ${ }^{9}$, A.Fenyuk ${ }^{41}$, P.Ferrari ${ }^{27}$, A.Ferrer ${ }^{48}$, S.Fichet $^{23}$, T.A.Filippas ${ }^{31}$, A.Firestone ${ }^{1}$, P.-A.Fischer ${ }^{10}$, H.Foeth ${ }^{9}$, E.Fokitis ${ }^{31}$, F.Fontanelli ${ }^{13}$, F.Formenti $^{9}$, B.Franek $^{36}$, A.G.Frodesen ${ }^{4}$, R.Fruhwirth ${ }^{49}$, F.Fulda-Quenzer ${ }^{19}$, J.Fuster ${ }^{48}$, A.Galloni ${ }^{22}$, D.Gamba ${ }^{44}$, M.Gandelman ${ }^{46}$, C.Garcia ${ }^{48}$, J.Garcia ${ }^{40}$, C.Gaspar $^{9}$, U.Gasparini ${ }^{35}$, Ph.Gavillet ${ }^{9}$, E.N.Gazis ${ }^{31}$, D.Gele ${ }^{10}$, J-P.Gerber ${ }^{10}$, R.Gokieli ${ }^{50}$, B.Golob ${ }^{42}$, P.Goncalves ${ }^{21}$, G.Gopal ${ }^{36}$, L.Gorn ${ }^{1}$, M.Gorski ${ }^{50}$, Yu.Gouz ${ }^{44,52}$, V.Gracco ${ }^{13}$, E.Graziani ${ }^{39}$, C.Green ${ }^{22}$, A.Grefrath ${ }^{51}$, P.Gris ${ }^{38}$, G.Grosdidier ${ }^{19}$, K.Grzelak ${ }^{50}$, S.Gumenyuk ${ }^{41}$, M.Gunther ${ }^{47}$, J.Guy ${ }^{36}$, F.Hahn ${ }^{9}$, S.Hahn ${ }^{51}$, Z.Hajduk ${ }^{18}$, A.Hallgren ${ }^{47}$, K.Hamacher ${ }^{51}$, F.J.Harris ${ }^{34}$, V.Hedberg $^{24}$, R.Henriques ${ }^{21}$, J.J.Hernandez ${ }^{48}$, P.Herquet ${ }^{2}$, H.Herr ${ }^{9}$, T.L.Hessing ${ }^{34}$, J.-M.Heuser ${ }^{51}$, E.Higon ${ }^{48}$, S-O.Holmgren ${ }^{43}$, P.J.Holt ${ }^{34}$, D.Holthuizen ${ }^{30}$, S.Hoorelbeke ${ }^{2}$, M.Houlden ${ }^{22}$, J.Hrubec ${ }^{49}$, K.Huet ${ }^{2}$, K.Hultqvist ${ }^{43}$, J.N.Jackson ${ }^{22}$, R.Jacobsson ${ }^{43}$, P.Jalocha ${ }^{9}$, R.Janik ${ }^{7}$, Ch.Jarlskog ${ }^{24}$, G.Jarlskog ${ }^{24}$, P.Jarry ${ }^{38}$, B.Jean-Marie ${ }^{19}$, E.K.Johansson ${ }^{43}$, L.Jonsson ${ }^{24}$, P.Jonsson ${ }^{24}$, C.Joram ${ }^{9}$, P.Juillot ${ }^{10}$, M.Kaiser ${ }^{17}$, F.Kapusta ${ }^{23}$, K.Karafasoulis ${ }^{11}{ }^{\text {, }}$, E.Karvelas $^{11}$, S.Katsanevas ${ }^{25}$, E.C.Katsoufis ${ }^{31}$, R.Keranen ${ }^{4}$, Yu.Khokhlov ${ }^{41}$, B.A.Khomenko ${ }^{16}$, N.N.Khovanski ${ }^{16}$, B.King ${ }^{22}$, N.J.Kjaer ${ }^{30}$, O.Klapp ${ }^{51}$, H.Klein ${ }^{9}$, P.Kluit ${ }^{30}$, D.Knoblauch ${ }^{17}$, P.Kokkinias ${ }^{11}$, M.Koratzinos $^{9}$, K.Korcyl ${ }^{18}$, V.Kostioukhine ${ }^{41}$, C.Kourkoumelis ${ }^{3}$, O.Kouznetsov ${ }^{16}$, M.Krammer ${ }^{49}$, C.Kreuter $^{9}$, I.Kronkvist $^{24}$, J.Krstic ${ }^{11}$, Z.Krumstein ${ }^{16}$, W.Krupinski ${ }^{18}$, P.Kubinec ${ }^{7}$, W.Kucewicz ${ }^{18}$, K.Kurvinen ${ }^{15}$, C.Lacasta $^{9}$, I.Laktineh ${ }^{25}$, J.W.Lamsa ${ }^{1}$, L.Lanceri ${ }^{45}$, D.W.Lane ${ }^{1}$, P.Langefeld ${ }^{51}$, V.Lapin ${ }^{41}$, J-P.Laugier ${ }^{38}$, R.Lauhakangas $^{15}$, G.Leder ${ }^{49}$, F.Ledroit ${ }^{14}$, V.Lefebure ${ }^{2}$, C.K.Legan ${ }^{1}$, A.Leisos ${ }^{11}$, R.Leitner ${ }^{29}$, J.Lemonne ${ }^{2}$, G.Lenzen $^{51}$, V.Lepeltier ${ }^{19}$, T.Lesiak ${ }^{18}$, M.Lethuillier $^{38}$, J.Libby ${ }^{34}$, D.Liko ${ }^{9}$, A.Lipniacka ${ }^{43}$, I.Lippi $^{35}$, B.Loerstad $^{24}$, J.G.Loken ${ }^{34}$, J.M.Lopez ${ }^{40}$, D.Loukas ${ }^{11}$, P.Lutz ${ }^{38}$, L.Lyons ${ }^{34}$, J.MacNaughton ${ }^{49}$, G.Maehlum ${ }^{17}$, J.R.Mahon $^{6}$, A.Maio ${ }^{21}$, T.G.M.Malmgren ${ }^{43}$, V.Malychev ${ }^{16}$, F.Mandl ${ }^{49}$, J.Marco ${ }^{40}$, R.Marco ${ }^{40}$, B.Marechal ${ }^{46}$, M.Margoni $^{35}$, J-C.Marin ${ }^{9}$, C.Mariotti ${ }^{9}$, A.Markou ${ }^{11}$, C.Martinez-Rivero ${ }^{33}$, F.Martinez-Vidal ${ }^{48}{ }^{\text {, }}$, S.Marti i Garcia ${ }^{22}$, J.Masik ${ }^{29}$, F.Matorras ${ }^{40}$, C.Matteuzzi ${ }^{27}$, G.Matthiae ${ }^{37}$, M.Mazzucato ${ }^{35}$, M.Mc Cubbin ${ }^{22}$, R.Mc Kay ${ }^{1}$, R.Mc Nulty ${ }^{9}$, G.Mc Pherson ${ }^{22}$, J.Medbo ${ }^{47}$, C.Meroni ${ }^{27}$, S.Meyer ${ }^{17}$, W.T.Meyer ${ }^{1}$, M.Michelotto ${ }^{35}$, E.Migliore $^{44}$, L.Mirabito ${ }^{25}$, U.Mjoernmark ${ }^{24}$, T.Moa ${ }^{43}$, R.Moeller ${ }^{28}$, K.Moenig ${ }^{9}$, M.R.Monge ${ }^{13}$, P.Morettini ${ }^{13}{ }^{\text {, }}$ H.Mueller $^{17}$, K.Muenich ${ }^{51}$, M.Mulders ${ }^{30}$, L.M.Mundim ${ }^{6}$, W.J.Murray ${ }^{36}$, B.Muryn ${ }^{14,18}$, G.Myatt ${ }^{34}$, T.Myklebust $^{32}$, F.Naraghi ${ }^{14}$, F.L.Navarria ${ }^{5}$, S.Navas $^{48}$, K.Nawrocki ${ }^{50}$, P.Negri ${ }^{27}$, S.Nemecek ${ }^{12}$, W.Neumann ${ }^{51}$, N.Neumeister $^{49}$, R.Nicolaidou ${ }^{3}$, B.S.Nielsen ${ }^{28}$, M.Nieuwenhuizen $^{30}$, V.Nikolaenko ${ }^{10}$, M.Nikolenko ${ }^{10,16}$, P.Niss ${ }^{43}$, A.Nomerotski $^{35}$, A.Normand ${ }^{22}$, A.Nygren ${ }^{24}$, W.Oberschulte-Beckmann ${ }^{17}$, V.Obraztsov ${ }^{41}$, A.G.Olshevski ${ }^{16}$, A.Onofre $^{21}$, R.Orava ${ }^{15}$, G.Orazi ${ }^{10}$, S.Ortuno ${ }^{48}$, K.Osterberg ${ }^{15}$, A.Ouraou ${ }^{38}$, P.Paganini ${ }^{19}$, M.Paganoni ${ }^{9,27}$, S.Paiano $^{5}$, R.Pain ${ }^{23}$, H.Palka ${ }^{18}$, Th.D.Papadopoulou ${ }^{31}$, K.Papageorgiou ${ }^{11}$, L.Pape ${ }^{9}$, C.Parkes ${ }^{34}$, F.Parodi $^{13}$, U.Parzefall $^{22}$, A.Passeri ${ }^{39}$, M.Pegoraro ${ }^{35}$, L.Peralta ${ }^{21}$, H.Pernegger ${ }^{49}$, M.Pernicka ${ }^{49}$, A.Perrotta ${ }^{5}$, C.Petridou $^{45}$, A.Petrolini $^{13}$, H.T.Phillips ${ }^{36}$, G.Piana ${ }^{13}$, F.Pierre ${ }^{38}$, M.Pimenta ${ }^{21}$, E.Piotto ${ }^{35}$, T.Podobnik ${ }^{34}$, O.Podobrin $^{9}$, M.E.Pol ${ }^{6}$, G.Polok ${ }^{18}$, P.Poropat ${ }^{45}$, V.Pozdniakov ${ }^{16}$, P.Privitera ${ }^{37}$, N.Pukhaeva ${ }^{16}$, A.Pullia ${ }^{27}$, D.Radojicic ${ }^{34}$, S.Ragazzi $^{27}$, H.Rahmani ${ }^{31}$, P.N.Ratoff ${ }^{20}$, A.L.Read ${ }^{32}$, M.Reale ${ }^{51}$, P.Rebecchi ${ }^{9}$, N.G.Redaelli ${ }^{27}$, M.Regler $^{49}$, D.Reid $^{9}$, R.Reinhardt ${ }^{51}$, P.B.Renton ${ }^{34}$, L.K.Resvanis ${ }^{3}$, F.Richard ${ }^{19}$, J.Ridky ${ }^{12}$, G.Rinaudo ${ }^{44}$, O.Rohne ${ }^{32}$, A.Romero $^{44}$, P.Ronchese ${ }^{35}$, L.Roos ${ }^{23}$, E.I.Rosenberg ${ }^{1}, \quad$ P.Rosinsky ${ }^{7}, \quad$ P.Roudeau $^{19}$, T.Rovelli ${ }^{5}$, V.Ruhlmann-Kleider ${ }^{38}$, A.Ruiz ${ }^{40}$, K.Rybicki ${ }^{18}$, H.Saarikko ${ }^{15}$, Y.Sacquin ${ }^{38}$, A.Sadovsky ${ }^{16}$, G.Sajot $^{14}$, J.Salt $^{48}$, M.Sannino $^{13}$, H.Schneider ${ }^{17}$, U.Schwickerath ${ }^{17}$, M.A.E.Schyns ${ }^{51}$, G.Sciolla ${ }^{44}$, F.Scuri ${ }^{45}$, P.Seager ${ }^{20}$, Y.Sedykh $^{16}{ }^{\text {, }}$, A.M.Segar ${ }^{34}$, A.Seitz ${ }^{17}$, R.Sekulin ${ }^{36}$, L.Serbelloni ${ }^{37}$, R.C.Shellard ${ }^{6}$, A.Sheridan ${ }^{22}$, P.Siegrist ${ }^{9,38}$, R.Silvestre ${ }^{38}$, F.Simonetto $^{35}$, A.N.Sisakian ${ }^{16}$, T.B.Skaali ${ }^{32}$, G.Smadja $^{25}, \quad$ N.Smirnov ${ }^{41}$, O.Smirnova ${ }^{24}$, G.R.Smith $^{36}$, A.Sokolov ${ }^{41}$, R.Sosnowski ${ }^{50}$, D.Souza-Santos ${ }^{6}$, T.Spassov ${ }^{21}$, E.Spiriti ${ }^{39}$, P.Sponholz ${ }^{51}$, S.Squarcia $^{13}$, D.Stampfer ${ }^{9}$, C.Stanescu ${ }^{39}$, S.Stanic ${ }^{42}$, S.Stapnes ${ }^{32}$, I.Stavitski ${ }^{35}$, K.Stevenson ${ }^{34}$, A.Stocchi ${ }^{19}$, J.Strauss ${ }^{49}$, R.Strub $^{10}$, B.Stugu ${ }^{4}$, M.Szczekowski ${ }^{50}$, M.Szeptycka ${ }^{50}$, T.Tabarelli ${ }^{27}$, J.P.Tavernet ${ }^{23}$, F.Tegenfeldt ${ }^{47}$, 
F.Terranova ${ }^{27}$, J.Thomas ${ }^{34}$, A.Tilquin ${ }^{26}$, J.Timmermans ${ }^{30}$, L.G.Tkatchev ${ }^{16}$, T.Todorov ${ }^{10}$, S.Todorova ${ }^{10}$, D.Z.Toet $^{30}$, A.Tomaradze ${ }^{2}$, B.Tome ${ }^{21}$, A.Tonazzo ${ }^{27}$, L.Tortora ${ }^{39}$, G.Transtromer ${ }^{24}$, D.Treille ${ }^{9}$, G.Tristram ${ }^{8}$, A.Trombini ${ }^{19}$, C.Troncon ${ }^{27}$, A.Tsirou ${ }^{9}, \quad$ M-L.Turluer ${ }^{38}$, I.A.Tyapkin ${ }^{16}$, M.Tyndel ${ }^{36}, \quad$ S.Tzamarias ${ }^{11}$, B.Ueberschaer ${ }^{51}$, O.Ullaland ${ }^{9}$, V.Uvarov ${ }^{41}$, G.Valenti ${ }^{5}$, E.Vallazza ${ }^{45}$, C.Vander Velde ${ }^{2}$, G.W.Van Apeldoorn ${ }^{30}$, P.Van Dam ${ }^{30}$, W.K.Van Doninck ${ }^{2}$, J.Van Eldik ${ }^{30}$, A.Van Lysebetten ${ }^{2}$, N.Vassilopoulos ${ }^{34}$, G.Vegni ${ }^{27}$, L.Ventura $^{35}$, W.Venus ${ }^{36}$, F.Verbeure ${ }^{2}$, M.Verlato ${ }^{35}$, L.S.Vertogradov ${ }^{16}$, D.Vilanova ${ }^{38}$, P.Vincent ${ }^{25}$, L.Vitale ${ }^{45}$, E.Vlasov $^{41}$, A.S.Vodopyanov ${ }^{16}$, V.Vrba ${ }^{12}$, H.Wahlen ${ }^{51}$, C.Walck ${ }^{43}$, F.Waldner ${ }^{45}$, C.Weiser ${ }^{17}$, A.M.Wetherell ${ }^{9}$, D.Wicke $^{51}$, J.H.Wickens ${ }^{2}$, M.Wielers ${ }^{17}$, G.R.Wilkinson ${ }^{9}$, W.S.C.Williams ${ }^{34}$, M.Winter ${ }^{10}$, M.Witek ${ }^{18}$, T.Wlodek $^{19}$, J.Yi $^{1}$, K.Yip $^{34}$, O.Yushchenko ${ }^{41}$, F.Zach $^{25}$, A.Zaitsev ${ }^{41}$, A.Zalewska ${ }^{9}$, P.Zalewski ${ }^{50}$, D.Zavrtanik ${ }^{42}$, E.Zevgolatakos ${ }^{11}$, N.I.Zimin ${ }^{16}$, G.C.Zucchelli ${ }^{43}$, G.Zumerle $^{35}$

\footnotetext{
${ }^{1}$ Department of Physics and Astronomy, Iowa State University, Ames IA 50011-3160, USA

${ }^{2}$ Physics Department, Univ. Instelling Antwerpen, Universiteitsplein 1, B-2610 Wilrijk, Belgium and IIHE, ULB-VUB, Pleinlaan 2, B-1050 Brussels, Belgium

and Faculté des Sciences, Univ. de l'Etat Mons, Av. Maistriau 19, B-7000 Mons, Belgium

${ }^{3}$ Physics Laboratory, University of Athens, Solonos Str. 104, GR-10680 Athens, Greece

${ }^{4}$ Department of Physics, University of Bergen, Allégaten 55, N-5007 Bergen, Norway

${ }^{5}$ Dipartimento di Fisica, Università di Bologna and INFN, Via Irnerio 46, I-40126 Bologna, Italy

${ }^{6}$ Centro Brasileiro de Pesquisas Físicas, rua Xavier Sigaud 150, RJ-22290 Rio de Janeiro, Brazil and Depto. de Física, Pont. Univ. Católica, C.P. 38071 RJ-22453 Rio de Janeiro, Brazil

and Inst. de Física, Univ. Estadual do Rio de Janeiro, rua São Francisco Xavier 524, Rio de Janeiro, Brazil

${ }^{7}$ Comenius University, Faculty of Mathematics and Physics, Mlynska Dolina, SK-84215 Bratislava, Slovakia

${ }^{8}$ Collège de France, Lab. de Physique Corpusculaire, IN2P3-CNRS, F-75231 Paris Cedex 05, France

${ }^{9}$ CERN, CH-1211 Geneva 23, Switzerland

${ }^{10}$ Institut de Recherches Subatomiques, IN2P3 - CNRS/ULP - BP20, F-67037 Strasbourg Cedex, France

${ }^{11}$ Institute of Nuclear Physics, N.C.S.R. Demokritos, P.O. Box 60228, GR-15310 Athens, Greece

${ }^{12}$ FZU, Inst. of Physics of the C.A.S. High Energy Physics Division, Na Slovance 2, 180 40, Praha 8, Czech Republic

${ }^{13}$ Dipartimento di Fisica, Università di Genova and INFN, Via Dodecaneso 33, I-16146 Genova, Italy

${ }^{14}$ Institut des Sciences Nucléaires, IN2P3-CNRS, Université de Grenoble 1, F-38026 Grenoble Cedex, France

${ }^{15}$ Helsinki Institute of Physics, HIP, P.O. Box 9, FIN-00014 Helsinki, Finland

${ }^{16}$ Joint Institute for Nuclear Research, Dubna, Head Post Office, P.O. Box 79, 101000 Moscow, Russian Federation

${ }^{17}$ Institut für Experimentelle Kernphysik, Universität Karlsruhe, Postfach 6980, D-76128 Karlsruhe, Germany

${ }^{18}$ Institute of Nuclear Physics and University of Mining and Metalurgy, Ul. Kawiory 26a, PL-30055 Krakow, Poland

${ }^{19}$ Université de Paris-Sud, Lab. de l'Accélérateur Linéaire, IN2P3-CNRS, Bât. 200, F-91405 Orsay Cedex, France

${ }^{20}$ School of Physics and Chemistry, University of Lancaster, Lancaster LA1 4YB, UK

${ }^{21}$ LIP, IST, FCUL - Av. Elias Garcia, 14-1 ${ }^{\circ}$, P-1000 Lisboa Codex, Portugal

${ }^{22}$ Department of Physics, University of Liverpool, P.O. Box 147, Liverpool L69 3BX, UK

${ }^{23}$ LPNHE, IN2P3-CNRS, Universités Paris VI et VII, Tour 33 (RdC), 4 place Jussieu, F-75252 Paris Cedex 05, France

${ }^{24}$ Department of Physics, University of Lund, Sölvegatan 14, S-22363 Lund, Sweden

${ }^{25}$ Université Claude Bernard de Lyon, IPNL, IN2P3-CNRS, F-69622 Villeurbanne Cedex, France

${ }^{26}$ Univ. d'Aix - Marseille II - CPP, IN2P3-CNRS, F-13288 Marseille Cedex 09, France

${ }^{27}$ Dipartimento di Fisica, Università di Milano and INFN, Via Celoria 16, I-20133 Milan, Italy

${ }^{28}$ Niels Bohr Institute, Blegdamsvej 17, DK-2100 Copenhagen 0, Denmark

${ }^{29}$ NC, Nuclear Centre of MFF, Charles University, Areal MFF, V Holesovickach 2, 180 00, Praha 8, Czech Republic

${ }^{30}$ NIKHEF, Postbus 41882, NL-1009 DB Amsterdam, The Netherlands

${ }^{31}$ National Technical University, Physics Department, Zografou Campus, GR-15773 Athens, Greece

${ }^{32}$ Physics Department, University of Oslo, Blindern, N-1000 Oslo 3, Norway

${ }^{33}$ Dpto. Fisica, Univ. Oviedo, Avda. Calvo Sotelo, S/N-33007 Oviedo, Spain, (CICYT-AEN96-1681)

${ }^{34}$ Department of Physics, University of Oxford, Keble Road, Oxford OX1 3RH, UK

${ }^{35}$ Dipartimento di Fisica, Università di Padova and INFN, Via Marzolo 8, I-35131 Padua, Italy

${ }^{36}$ Rutherford Appleton Laboratory, Chilton, Didcot OX11 OQX, UK

${ }^{37}$ Dipartimento di Fisica, Università di Roma II and INFN, Tor Vergata, I-00173 Rome, Italy

${ }^{38} \mathrm{CEA}$, DAPNIA/Service de Physique des Particules, CE-Saclay, F-91191 Gif-sur-Yvette Cedex, France

${ }^{39}$ Istituto Superiore di Sanità, Ist. Naz. di Fisica Nucl. (INFN), Viale Regina Elena 299, I-00161 Rome, Italy

${ }^{40}$ Instituto de Fisica de Cantabria (CSIC-UC), Avda. los Castros, S/N-39006 Santander, Spain, (CICYT-AEN96-1681)

${ }^{41}$ Inst. for High Energy Physics, Serpukov P.O. Box 35, Protvino, (Moscow Region), Russian Federation

42 J. Stefan Institute, Jamova 39, SI-1000 Ljubljana, Slovenia and Department of Astroparticle Physics, School of Environmental Sciences, Kostanjeviska 16a, Nova Gorica, SI-5000 Slovenia, and Department of Physics, University of Ljubljana, SI-1000 Ljubljana, Slovenia

${ }^{43}$ Fysikum, Stockholm University, Box 6730, S-113 85 Stockholm, Sweden

${ }^{44}$ Dipartimento di Fisica Sperimentale, Università di Torino and INFN, Via P. Giuria 1, I-10125 Turin, Italy

${ }^{45}$ Dipartimento di Fisica, Università di Trieste and INFN, Via A. Valerio 2, I-34127 Trieste, Italy and Istituto di Fisica, Università di Udine, I-33100 Udine, Italy

${ }^{46}$ Univ. Federal do Rio de Janeiro, C.P. 68528 Cidade Univ., Ilha do Fundão BR-21945-970 Rio de Janeiro, Brazil

${ }^{47}$ Department of Radiation Sciences, University of Uppsala, P.O. Box 535, S-751 21 Uppsala, Sweden

${ }^{48}$ IFIC, Valencia-CSIC, and D.F.A.M.N., U. de Valencia, Avda. Dr. Moliner 50, E-46100 Burjassot (Valencia), Spain

${ }^{49}$ Institut für Hochenergiephysik, Österr. Akad. d. Wissensch., Nikolsdorfergasse 18, A-1050 Vienna, Austria

${ }^{50}$ Inst. Nuclear Studies and University of Warsaw, Ul. Hoza 69, PL-00681 Warsaw, Poland

${ }^{51}$ Fachbereich Physik, University of Wuppertal, Postfach 100 127, D-42097 Wuppertal, Germany

${ }^{52}$ On leave of absence from IHEP Serpukhov
} 


\section{Introduction}

In 1996, the LEP centre-of-mass energy reached $172 \mathrm{GeV}$, and the DELPHI experiment collected integrated luminosities of $9.7 \mathrm{pb}^{-1}$ and $10.0 \mathrm{pb}^{-1}$ at $161 \mathrm{GeV}$ and $172 \mathrm{GeV}$, respectively. These data have been analysed to search for the supersymmetric partners of Higgs and gauge bosons, the charginos, neutralinos and gravitinos, predicted by supersymmetric (SUSY) models [1].

The methods used to search for charginos and neutralinos presented in [2-5] have remained almost unchanged. Minor modifications have been introduced in order to reject the additional $W$ pair background present at the higher energies, and to scale certain of the energy, momentum and mass values used in the selection criteria in proportion to the centre-of-mass energy.

The conservation of R-parity, implying a stable lightest supersymmetric particle (LSP), is also assumed in the present paper. While the previous papers assumed the LSP to be the lightest neutralino $\left(\tilde{\chi}_{1}^{0}\right)$, the present analysis also treats the case where the LSP is a very light gravitino $(G)$. In the former case, events are characterised by missing momentum carried by the escaping neutralinos, while in the latter case the decay $\tilde{\chi}_{1}^{0} \rightarrow \tilde{\mathrm{G}} \gamma$ is possible [6-8]. If the gravitino is sufficiently light (with a mass below about $250 \mathrm{eV} / \mathrm{c}^{2}$ [8]), this decay takes place within the detector. As gravitinos escape detection, the typical signature of these SUSY events is missing momentum and isolated photons. Unless the contrary is explicitly stated, the Minimal Supersymmetric Standard Model (MSSM) scheme with universal parameters at the high mass scale typical of Grand Unified Theories (GUT's) is assumed [1]. The parameters of this model relevant to the present searches are the masses $M_{1}$ and $M_{2}$ of the gaugino sector (which are assumed to satisfy the GUT relation $M_{1}=\frac{5}{3} \tan ^{2} \theta_{W} M_{2} \approx 0.5 M_{2}$ at the electroweak scale), the universal mass $m_{0}$ of the scalar lepton sector (which enters mainly via the sneutrino mass), the Higgs mass parameter $\mu$, and the ratio $\tan \beta$ of the vacuum expectation values of the two Higgs doublets. In this scheme, the chargino production cross-section at a given energy can be greatly reduced by destructive interference between the $s$-channel and $t$-channel contributions if the sneutrino mass is below $300 \mathrm{GeV} / c^{2}$ and the SUSY parameters take particular values $[9]$.

\section{Detector description}

DELPHI is a general purpose detector with a magnetic field of $1.2 \mathrm{~T}$ provided by a large superconducting solenoid. The detector and its performance are described in [10].

The main tracking device is the Time Projection Chamber (TPC). Other tracking devices used to reconstruct charged particle tracks at large angles with respect to the beam axis ('barrel region') are the Vertex Detector, the Inner Detector (ID) and the Outer Detector. For particles emerging at smaller angles ('forward region'), the forward drift chambers (FCA and FCB) supplement the TPC and the ID for track reconstruction.

The electromagnetic calorimeters in the forward region are the Forward Electromagnetic Calorimeter (FEMC), an array of lead glass blocks covering the polar angular regions $8^{\circ}<\theta<35^{\circ}$ and $145^{\circ}<\theta<172^{\circ}$, and the STIC, a sampling electromagnetic calorimeter which covers the angular regions $1.7^{\circ}<\theta<10.6^{\circ}$ and $169.4^{\circ}<\theta<178.3^{\circ}$. The High density Projection Chamber (HPC), the electromagnetic calorimeter in the barrel, covers the angular range $43.1^{\circ}<\theta<88.7^{\circ}$ and $91.3^{\circ}<\theta<136.9^{\circ}$. The HPC has a total of 144 modules, each radially segmented into 9 layers. 
Photon detection in the regions between the electromagnetic calorimeters is achieved using information from dedicated taggers and other detectors. The $40^{\circ}$ taggers are a series of photon counters, each consisting of $2 \mathrm{~cm}$ of lead followed by a layer of scintillator, used to veto photons that could otherwise be missed in the $\theta$ regions near $40^{\circ}$ and $140^{\circ}$ between the HPC and FEMC. Similar taggers are installed at polar angles $88^{\circ}-92^{\circ}$, between the two halves of the HPC. The Time of Flight detector (TOF), consisting of a single layer of 172 scintillation counters just outside the solenoid and covering the polar angular region $41^{\circ}<\theta<139^{\circ}$, helps to increase the hermeticity in the regions in azimuthal angle between the modules of the HPC. The few azimuthal regions not covered by the TOF are equipped with another set of counters similar to the $40^{\circ}$ taggers.

The hadron calorimeter (HCAL) is segmented radially into 4 layers and covers $98 \%$ of the solid angle. Muon identification uses chambers placed between the third and the fourth HCAL layer and outside the fourth layer, covering nearly all the solid angle.

\section{Data samples and event generators}

The data samples accumulated at centre-of-mass energies of $161 \mathrm{GeV}$ and $172 \mathrm{GeV}$ were used in the search for chargino and neutralino production as described in sections 4.1 to 4.6 . The 1995 data $[2,5,11]$ amounting to $2.92 \mathrm{pb}^{-1}$ and $3.01 \mathrm{pb}^{-1}$ at centre-ofmass energies of $130.4 \mathrm{GeV}$ and $136.3 \mathrm{GeV}$ respectively, were also used in the search for charginos and neutralinos in the scenario where $\tilde{\chi}_{1}^{0} \rightarrow \tilde{G} \gamma$. The results obtained at the $\mathrm{Z}$ resonance were also included in the search for $\tilde{\chi}_{1}^{0} \tilde{\mathrm{G}}$ production. To evaluate the signal efficiencies and background contaminations, events were generated using several different programs. All relied on JETSET 7.4 [12], tuned to LEP 1 data [13], for quark fragmentation.

The program SUSYGEN [14] was used to generate chargino and neutralino signal events and to calculate cross-sections and branching ratios. These agree with the calculations of Ref. [15]. A modified version of the same program was used to generate signal events in the scenario with a light gravitino.

The background process $\mathrm{e}^{+} \mathrm{e}^{-} \rightarrow \mathrm{q} \overline{\mathrm{q}}(n \gamma)$ was generated with PYTHIA 5.7 [12], while DYMU3 [16] and KORALZ [17] were used for $\mu^{+} \mu^{-}(\gamma)$ and $\tau^{+} \tau^{-}(\gamma)$, respectively. The generator of Ref. [18] was used for $\mathrm{e}^{+} \mathrm{e}^{-} \rightarrow \mathrm{e}^{+} \mathrm{e}^{-}$events.

Processes leading to four-fermion final states, $(\mathrm{Z} / \gamma)^{*}(\mathrm{Z} / \gamma)^{*}, \mathrm{~W}^{+} \mathrm{W}^{-}, \mathrm{We} \nu_{\mathrm{e}}$ and $\mathrm{Ze}^{+} \mathrm{e}^{-}$, were also generated using PYTHIA. The calculation of the four-fermion background was verified using the program EXCALIBUR [19], which consistently takes into account all amplitudes leading to a given four-fermion final state. EXCALIBUR does not, however, include the transverse momentum of initial state radiation.

Two-photon interactions leading to hadronic final states were generated using TWOGAM [20], separating the VDM, QPM, and QCD components. The generators of Berends, Daverveldt and Kleiss [21] were used for the leptonic final states.

The generated signal and background events were passed through the detailed simulation of the DELPHI detector [10] and then processed with the same reconstruction and analysis programs as the real data. The simulated number of events from different background processes was several times the number in the real data; details of the signal samples generated are given in sections 5,6 . 


\section{Event selections}

The criteria used to select events were defined on the basis of the simulated signal and background events. The selections for charged and neutral particles were similar to those presented in $[2,3,5]$ requiring charged particles to have momentum above $100 \mathrm{MeV} / \mathrm{c}$ and to extrapolate back to within $5 \mathrm{~cm}$ of the main vertex in the transverse plane, and to within twice this distance in the longitudinal direction. Calorimeter energy clusters above $100 \mathrm{MeV}$ were taken as neutral particles if not associated to any charged particle track. The particle selection was followed by different event selections for the different signal topologies.

\section{Topologies with a stable neutralino}

For $\tilde{\chi}_{1}^{+} \tilde{\chi}_{1}^{-}$chargino pair production with $\tilde{\chi}_{1}^{ \pm} \rightarrow \tilde{\chi}_{1}^{0} f_{f^{\prime}}$, the relevant visible topologies with a stable neutralino are $j j \ell$ (two jets, one isolated lepton, and missing momentum), jets (multijet events with missing momentum), and $\ell \ell$ (two leptons and missing momentum). The search for neutralino production in the case of a stable $\tilde{\chi}_{1}^{0}$ concentrated on the most significant visible final states, $\tilde{\chi}_{1}^{0} \tilde{\chi}_{2}^{0}, \tilde{\chi}_{1}^{0} \tilde{\chi}_{3}^{0}$ and $\tilde{\chi}_{1}^{0} \tilde{\chi}_{4}^{0}$, with $\tilde{\chi}_{k}^{0} \rightarrow \tilde{\chi}_{1}^{0}+\mathrm{ff} ; k=2,3,4$. These lead to $j j$ events (two jets and missing momentum) and $\ell \ell$ events. A characteristic feature of all of these topologies is the presence of jets or leptons which are acoplanar with the beam, missing momentum transverse to the beam $\left(p_{T}^{\text {miss }}\right)$, and missing mass due to escaping neutralinos. The distribution of $\cos \theta$, where $\theta$ is the polar angle of a jet or lepton, is nearly uniform. In addition to the neutralino $\tilde{\chi}_{1}^{0} f \bar{f}$ modes, DELPHI results on single-photon production at LEP 1 [22] were interpreted as limits on $Z \rightarrow \tilde{\chi}_{1}^{0} \tilde{\chi}_{2}^{0} \rightarrow \tilde{\chi}_{1}^{0} \tilde{\chi}_{1}^{0} \gamma$.

The mass difference $\Delta M=M_{\tilde{\chi}_{1}^{ \pm}}-M_{\tilde{\chi}_{1}^{0}}$ in $\tilde{\chi}_{1}^{+} \tilde{\chi}_{1}^{-}$events or $\Delta M=M_{\tilde{\chi}_{k}^{0}}-M_{\tilde{\chi}_{1}^{0}}$ in $\tilde{\chi}_{k}^{0} \tilde{\chi}_{1}^{0}$ events, plays an important rôle as the typical $p_{T}^{\text {miss }}$ and visible mass grow with $\Delta M$. For low $\Delta M$ the major background consists of two-photon events, whereas $\mathrm{W}^{+} \mathrm{W}^{-}$and $\mathrm{f} \bar{f}$ events with initial state radiation are the background for large $\Delta M$. Several analyses were performed to optimise the selection for different ranges of $\Delta M$. The range of accessible mass differences is larger in the neutralino case than in the chargino case, which leads to different analyses for neutralinos and charginos. Note also that the chargino jets events appear as four-jet events for high $\Delta M$ and as two-jet events for low $\Delta M$, whereas the two jets in neutralino $j j$ events tend to merge for low $\Delta M$. The LUCLUS algorithm [12] was used to reconstruct jets in both chargino and neutralino searches. In the chargino search a forced two jet reconstruction was applied. In the neutralino search it was required that at least two jets were reconstructed and that there were no more than two jets with a minimum transverse jet separation, $d_{\text {join }}=10 \mathrm{GeV} / c$. In chargino production an additional complication may arise if there is a sneutrino lighter than the chargino in which case the decay $\tilde{\chi}_{1}^{+} \rightarrow \tilde{\nu} \ell$ dominates (with $\tilde{\nu}$ decaying invisibly). The relevant $\Delta M$ is then $M_{\tilde{\chi}_{1}^{ \pm}}-M_{\tilde{\nu}}$, but because of the two-body decay the sensitivity to low $\Delta M$ is actually higher than for $\tilde{\chi}_{1}^{+} \rightarrow \tilde{\chi}_{1}^{0} \nu \ell$.

Two-photon events are characterised by large missing momentum and energy, and low visible mass. However, $p_{T}^{\text {miss }}$ is small, the jet or lepton system tends to be coplanar with the beam direction, and there are often jets in the forward-backward region of the detector. These events can thus be rejected by requiring significant transverse momentum and acoplanarity and a small jet activity in the forward-backward region. In the hadronic topologies ( $j j, j j \ell$, and jets), the 'scaled acoplanarity' was used in the selection. This is the acoplanarity between the planes containing each jet and the beam, when exactly two jets were reconstructed with the LUCLUS algorithm [12], multiplied by the lower value of $\sin \theta_{\text {jet }}$ to account for the worse definition of acoplanarity for forward jets. 
In $f \bar{f}$ events with initial state radiation ('radiative return to the $\mathrm{Z}$ ') there is a high energy photon which is usually lost in the beam pipe. Such events are characterised by large visible mass $\left(\sim M_{\mathrm{Z}}\right)$ and large missing momentum aligned with the beam direction. They may be rejected by requiring significant $p_{T}^{\text {miss }}$ or acoplanarity. For $\tilde{\chi}_{k}^{0} \tilde{\chi}_{1}^{0}$ there is a loss of efficiency when $\Delta M=M_{\tilde{\chi}_{k}^{0}}-M_{\tilde{\chi}_{1}^{0}}=M_{\mathrm{Z}}$, because of the need to reject this particular background. The background from $\mathrm{ff}$ events with initial state radiation emitted at larger polar angles was reduced by vetoing events with energetic isolated photons in the detector. In this case both the acoplanarity and the $p_{\mathrm{T}}$ of the $\mathrm{f} \bar{f}$ system can be large and radiative events with photons missing the electromagnetic calorimeters were a potentially serious background. To reduce it, events were rejected if the hermeticity detectors gave a signal which coincided in azimuth with the missing momentum. Using data taken at the Z resonance (LEP 1 data), it has been shown [5] that the total photon detection efficiency of DELPHI is above $99 \%$ for photons of more than $5 \mathrm{GeV}$ in the range $20^{\circ}<\theta<160^{\circ}$.

$\mathrm{W}^{+} \mathrm{W}^{-}$events constitute a practically irreducible background to the $\tilde{\chi}_{1}^{+} \tilde{\chi}_{1}^{-}$topologies listed above when $\Delta M=M_{\mathrm{W}}$. For large $\Delta M<M_{\mathrm{W}}$ there is a loss of efficiency for these topologies because of the need to reject $\mathrm{W}^{+} \mathrm{W}^{-}$background. For large $\Delta M$, however, the charginos tend to decay via $\tilde{\chi}_{1}^{ \pm} \rightarrow \tilde{\chi}_{2}^{0} \mathrm{ff} \bar{f}^{\prime} \rightarrow \tilde{\chi}_{1}^{0} \gamma f \bar{f}^{\prime}$ so the $\mathrm{W}^{+} \mathrm{W}^{-}$background can be reduced by requiring the presence of photons (see Topologies with an unstable neutralino, below).

Fig. 1 shows the distribution of variables relevant for the selection of topologies with a stable neutralino, namely multiplicity, visible mass, and acoplanarity, for real and simulated events. The distributions to the left in Fig. 1 ('leptonic selection') refer to events selected by requiring a pair of oppositely charged particles with transverse momentum $p_{T}^{\text {pair }}>3 \mathrm{GeV} / c$. The total number of reconstructed charged particle tracks had to be below ten, and the energy in the forward-backward $30^{\circ}$ cones had to be less than half the visible energy. The 'hadronic selection' (right in figure) required five or more charged particles with a total energy above $4 \mathrm{GeV}, p_{T}^{\text {miss }}>3 \mathrm{GeV} / c$, and the same requirement on the energy in the forward-backward regions. The agreement between real and simulated data is reasonable; the normalization is absolute.

Topologies with an unstable neutralino

If the lightest neutralino is not stable but decays within the detector into a photon and a gravitino, then the $\tilde{\chi}_{1}^{+} \tilde{\chi}_{1}^{-}$topologies described above change by the addition of isolated energetic photons coming from the neutralino decay. Searches for such events were conducted in the chargino channels (the $\gamma \gamma \mathrm{X}$ topologies, where $\mathrm{X}$ stands for $j j \ell$, jets or $\ell \ell$ ) in a way similar to the search in the stable neutralino scenario. In this case, however, events with isolated photons were not rejected. Instead, the presence of at least one isolated photon was required in the selection criteria, as described in section 4.4.

In the search for neutralinos in the case of an unstable $\tilde{\chi}_{1}^{0}$ decaying into $\gamma \tilde{\mathrm{G}}$, different processes than in the stable neutralino case were considered, namely $\mathrm{e}^{+} \mathrm{e}^{-} \rightarrow \tilde{\chi}_{1}^{0} \tilde{\mathrm{G}}$ and $\mathrm{e}^{+} \mathrm{e}^{-} \rightarrow \tilde{\chi}_{1}^{0} \tilde{\chi}_{1}^{0}$. These would produce, respectively, a single-photon or two acoplanar photons in the final state and will be referred to as single- $\gamma$ and $\gamma \gamma$ topologies in the following. The reaction $\mathrm{e}^{+} \mathrm{e}^{-} \rightarrow \tilde{\chi}_{1}^{0} \tilde{\mathrm{G}}$ derives from the possibility of direct $\tilde{\chi}_{1}^{0} \tilde{\mathrm{G}} \gamma$ and $\tilde{\chi}_{1}^{0} \tilde{\mathrm{G} Z}$ couplings [6], which are also responsible for the $\tilde{\chi}_{1}^{0}$ decay.

\subsection{Chargino decays giving $j j \ell$ final states}

Two different sets of selection criteria were used, optimised for different ranges of the mass difference between the chargino and the lightest neutralino, $\Delta M$. 
In the 'non-degenerate' case, $\Delta M>10 \mathrm{GeV} / c^{2}$, events were required to have at least three charged particles, a total multiplicity of at least 10 and a scaled acoplanarity of at least $15^{\circ}$. The presence of a loosely identified electron or muon [10] of at least $3 \mathrm{GeV} / \mathrm{c}$ was required. This lepton had to be emitted at an angle of at least $20^{\circ}$ from both jets. To reduce the $\mathrm{W}^{+} \mathrm{W}^{-}$background, events with an isolated lepton with a momentum greater than $30 \mathrm{GeV} / \mathrm{c}$ were rejected. Moreover, the events were required to have either an invariant mass $M_{j j}$ of the hadronic system (excluding the lepton) smaller than $40 \mathrm{GeV} / c^{2}$, or an invariant mass $M_{l \nu}$ reconstructed using the lepton and the missing momentum (assumed to be due to an unseen neutrino) smaller than $60 \times\left(1-\frac{M_{j j}}{65}\right) \mathrm{GeV} / c^{2}$. The missing transverse momentum of the event was required to be larger than $4 \mathrm{GeV} / \mathrm{c}$. Furthermore, in order to reduce the two-photon background, events were rejected if more than $50 \%$ of the visible energy was contained within the forward and backward $20^{\circ}$ cones around the beam. To reject $\mathrm{e}^{+} \mathrm{e}^{-} \rightarrow \mathrm{e}^{+} \mathrm{e}^{-}$events the electromagnetic energy was required to be smaller than $90 \%$ of the visible energy.

For the 'degenerate' case, $\Delta M \leq 10 \mathrm{GeV} / c^{2}$, the requirement of a minimum total multiplicity was removed, the minimum momentum of the isolated lepton was reduced to $1 \mathrm{GeV} / c$, and the minimum missing transverse momentum with respect to the beam axis to $3 \mathrm{GeV} / c$. In order to compensate for the loss of purity caused by relaxing these criteria, the maximum allowed energy in the forward and backward $20^{\circ}$ cones was reduced to $40 \%$ of the visible energy, and events with a visible energy larger than $30 \mathrm{GeV}$ or with an isolated lepton with a momentum greater than $20 \mathrm{GeV} / c$ were rejected.

\subsection{Chargino decays giving jets final states}

In the non-degenerate case $\left(\Delta M>10 \mathrm{GeV} / c^{2}\right)$, events were selected by requiring charged and total multiplicities of at least 3 and 10 respectively, and the absence of isolated leptons as defined in section 4.1. Bhabha events were rejected by requiring the electromagnetic energy to be smaller than $90 \%$ of the visible energy. The scaled acoplanarity was required to be greater than $20^{\circ}$, while both jet axes had to point in the polar angular region $24^{\circ}<\theta<156^{\circ}$. The energy in the forward and backward $20^{\circ}$ cones was required to be below $30 \%$ of the visible energy. The missing transverse momentum of the event (relative to the beam axis) had to be above $5 \mathrm{GeV} / c$ and the polar angle of the missing momentum had to be in the region $30^{\circ}<\theta_{p_{\text {miss }}}<150^{\circ}$. Finally, a total visible mass below $68 \mathrm{GeV} / \mathrm{c}^{2}$ was required.

In the degenerate case $\left(\Delta M \leq 10 \mathrm{GeV} / c^{2}\right)$, events were required to have at least five charged particles, with no additional requirement of a minimum total multiplicity. There should be no isolated lepton with momentum above $3 \mathrm{GeV} / c$. The scaled acoplanarity had to be above $40^{\circ}$. The energy in the forward and backward $20^{\circ}$ cones had to be below $25 \%$ of the visible energy. The missing transverse momentum of the event had to be above $5 \mathrm{GeV} / c$ and the polar angle of the missing momentum had to be in the region $30^{\circ}<\theta_{p_{\text {miss }}}<150^{\circ}$. The energy deposited in the electromagnetic calorimeters had to be below $90 \%$ of the visible energy, and the visible mass below $25 \mathrm{GeV} / \mathrm{c}^{2}$.

\subsection{Chargino decays giving $\ell \ell$ final states}

The first steps in the selection were identical for the degenerate and non-degenerate cases. At least two well reconstructed charged particle tracks originating from the vertex region were required and the total multiplicity had to be eight or less. If neither of the two most energetic charged particles had associated hits in the muon chambers the 
difference in $\theta$ between these two particles had to be at least $5^{\circ}$. The energy detected in the electromagnetic and hadronic calorimeters had to be less than $50 \mathrm{GeV}$ and $40 \mathrm{GeV}$, respectively, and the energy in the forward and backward $30^{\circ}$ cones was required to be below $50 \%$ of the visible energy. The visible energy was required to be less than $62.5 \mathrm{GeV}$ and $12 \mathrm{GeV}$ in the non-degenerate and degenerate cases, respectively, and in the degenerate case the visible mass was required to be less than $8 \mathrm{GeV}$.

In order to reduce the $W$ pair background, the most energetic well reconstructed particle originating from the vertex region was required to have a momentum below $30 \mathrm{GeV} / c$ and $6 \mathrm{GeV} / c$ in the non-degenerate case and degenerate case, respectively.

Background $\gamma \gamma \rightarrow \tau^{+} \tau^{-}$events with 1-prong decays of the $\tau^{\prime}$ 's give two nearly backto-back tracks in the transverse plane because of the low $Q$-value of the $\tau$ decay. To reject such background, the momenta of the two most energetic particles were projected onto the plane transverse to the beam. The projected momenta were used to define a thrust axis, and their vector sum perpendicular to this axis was calculated. Its square was required to exceed $0.4(\mathrm{GeV} / \mathrm{c})^{2}$.

In the case of more than two charged particles, the selection was completed by requirements on acoplanarity, visible mass, and missing transverse momentum. The acoplanarity of the two most energetic particles was required to be between $10^{\circ}$ and $160^{\circ}$ and the visible mass had to be greater than $4 \mathrm{GeV} / c^{2}$. The missing transverse momentum, finally, was required to exceed $5 \mathrm{GeV} / c$ in the non-degenerate case and $4 \mathrm{GeV} / c$ in the degenerate case.

In the case of exactly two charged particles, the acoplanarity had to be at least $20^{\circ}$, and the azimuthal angle of the missing momentum had to be outside $\pm 5^{\circ}$ of any energy deposit in the STIC. This was to reject background arising from the poor energy measurement for low-angle hadrons in that detector. The invariant mass of the two charged particles had also to be above $4 \mathrm{GeV} / \mathrm{c}^{2}$ and the missing transverse momentum above $5 \mathrm{GeV} / \mathrm{c}$. The momentum of the second most energetic particle was required to be greater than $0.5 \mathrm{GeV} / \mathrm{c}$ and the energy in the forward and backward $30^{\circ}$ cones had to be smaller than $20 \%$ of the visible energy.

\subsection{Chargino production giving $\gamma \gamma \mathrm{X}$ final states}

A special analysis was developed to search for an unstable $\tilde{\chi}_{1}^{0}$, decaying into a photon and a light gravitino. The selection is also sensitive to cascade decays of $\tilde{\chi}_{1}^{ \pm}$into $\tilde{\chi}_{2}^{0}$ which in turn decays into $\tilde{\chi}_{1}^{0} \gamma$ where $\tilde{\chi}_{1}^{0}$ is stable. Since the presence of one or two isolated photons is a very discriminating signal, no distinction was made between the different chargino decay topologies $(j j \ell$, jets, and $\ell \ell)$. In a first step, events were selected if they contained at least one charged particle and at least one energetic isolated photon and

- the photon polar angles, $\theta_{\gamma}$, satisfied $15^{\circ}<\theta_{\gamma}<165^{\circ}$ and the photon energies exceeded $5 \mathrm{GeV}$,

- the angles of isolation between each photon and any other well reconstructed neutral or charged particle were greater than $15^{\circ}$.

In the non-degenerate case, the total visible mass had to be smaller than $130 \mathrm{GeV} / \mathrm{c}^{2}$, the energy of the most energetic isolated photon smaller than $45 \mathrm{GeV}$, and the momentum of the most energetic charged particle smaller than $30 \mathrm{GeV} / c$. Then, different selection criteria were applied depending on whether one photon or two (or more) isolated photons were detected. In the latter case, the only further requirement was that the scaled 
acoplanarity had to be greater than $5^{\circ}$. If only one isolated photon was detected, the scaled acoplanarity had to be greater than $15^{\circ}$, the missing transverse momentum greater than $5 \mathrm{GeV} / c$, and the electromagnetic energy less than $90 \%$ of the total calorimetric energy.

In the degenerate case, the missing transverse momentum had to be greater than $5 \mathrm{GeV} / c$, the scaled acoplanarity greater than $5^{\circ}$, and the polar angle of the missing momentum had to satisfy $10^{\circ}<\theta_{p_{m i s s}}<170^{\circ}$. The momentum of the most energetic charged particle was required to be smaller than $30 \mathrm{GeV} / c$. In addition, the energy of the most energetic isolated photon, as defined above, had to lie between $15 \mathrm{GeV}$ and $45 \mathrm{GeV}$. The visible mass of the event, excluding the isolated photons, had to be smaller than $50 \mathrm{GeV} / \mathrm{c}^{2}$. The percentage of energy in the forward and backward $30^{\circ}$ cones had to be smaller than $70 \%$ and the total electromagnetic energy, excluding the energy of the most energetic isolated photon, had to be below $70 \mathrm{GeV}$.

\subsection{Neutralino decays giving $j j$ final states}

In order to select hadronic events at least five particles were required, and at least two of these had to be charged. Two jets were required, each satisfying $20^{\circ}<\theta_{\text {jet }}<160^{\circ}$ and containing at least one charged particle reconstructed with small momentum error. To reject background from $\mathrm{W}^{+} \mathrm{W}^{-}$production, no isolated charged particle with energy greater than $3 \mathrm{GeV}$ and at an angle of more than $10^{\circ}$ away from the nearest jet was allowed.

To reject $\mathrm{Z} \gamma$ and two-photon background, several criteria were used. The total energy of particles emitted within $30^{\circ}$ of the beam was required to be smaller than $40 \%$ of the total visible energy, the polar angle of the missing momentum had to satisfy $20^{\circ}<$

$\theta p_{\text {miss }}<160^{\circ}$, and the missing transverse momentum had to exceed $5.5 \mathrm{GeV} / c$. The invariant mass of visible particles was required to be less than $0.55 \sqrt{s} / c^{2}$, and the missing mass to exceed $0.25 \sqrt{s} / c^{2}$. The scaled acoplanarity had to exceed $10^{\circ}$.

In addition, events were required to satisfy at least one of the following three sets of criteria, sensitive to different ranges of $\Delta M=M_{\tilde{\chi}_{2}^{0}}-M_{\tilde{\chi}_{1}^{0}}$ :

- Low $\Delta M\left(\sim 10 \mathrm{GeV} / c^{2}\right)$ : The invariant mass of visible particles was required to be less than $0.1 \sqrt{s} / c^{2}$, the missing mass had to exceed $0.7 \sqrt{s} / c^{2}$, and the scaled acoplanarity had to be greater than $40^{\circ}$.

- Intermediate $\Delta M\left(\sim 40 \mathrm{GeV} / c^{2}\right)$ : More than six particles were required. The invariant mass of visible particles was required to be less than $0.4 \sqrt{s} / c^{2}$, and the missing mass had to exceed $0.5 \sqrt{s} / c^{2}$. The total energy of particles in the forward and backward $30^{\circ}$ cones was required to be smaller than $25 \%$ of the total visible energy. The polar angle of missing momentum should satisfy $30^{\circ}<\theta_{p_{\text {miss }}}<150^{\circ}$. In addition, the scaled acoplanarity had to exceed $30^{\circ}$ and missing transverse momentum had to exceed $7.5 \mathrm{GeV} / c$.

- Large $\Delta M\left(\sim 90 \mathrm{GeV} / c^{2}\right)$ : More than eight particles were required, the polar angle of the missing momentum was required to satisfy $30^{\circ}<\theta_{p_{\text {miss }}}<150^{\circ}$, and the missing transverse momentum to be greater than $15 \mathrm{GeV} / \mathrm{c}$.

\subsection{Neutralino decays giving $\ell \ell$ final states}

To select the di-lepton topology, two and only two isolated, oppositely charged, particles with momenta above $1 \mathrm{GeV} / c$, relative momentum error smaller than $30 \%$, and polar angles in the range $20^{\circ}<\theta<160^{\circ}$ were required. One particle or both had to be at 
least loosely identified as an electron or muon according to [10], and no more than $40 \%$ of the centre-of-mass energy was allowed to be associated with either particle. Events with more than three charged particles with momenta greater than $1 \mathrm{GeV} / c$ were rejected. The total energy of particles in the forward and backward $30^{\circ}$ cones had to be less than $30 \%$ of the visible energy, and the polar angle of the missing momentum had to satisfy $20^{\circ}<\theta_{p_{\text {miss }}}<160^{\circ}$. To reject $\mathrm{e}^{+} \mathrm{e}^{-} \rightarrow \mathrm{e}^{+} \mathrm{e}^{-}$events, the acoplanarity between the two selected particles was required to exceed $10^{\circ}$.

In order to reject background from $\mathrm{e}^{+} \mathrm{e}^{-} \rightarrow \mathrm{q} \overline{\mathrm{q}}(\gamma)$ events and two-photon interactions, the total multiplicity of the event was required to be seven or smaller and the energy deposited in the STIC had to be less than $12 \mathrm{GeV}$.

Requirements for different missing energy regions were optimised for different $M_{\tilde{\chi}_{2}^{0}}-$ $M_{\tilde{\chi}_{1}^{0}}$. If the missing energy of the event was greater than $130 \mathrm{GeV}$, the two particles were required to have a total transverse momentum above $5.5 \mathrm{GeV} / c$, and an acoplanarity in excess $15^{\circ}$. If the missing energy was between $0.3 \sqrt{s}$ and $130 \mathrm{GeV}$, the transverse momentum had to be at least $7 \mathrm{GeV} / c$ while the minimum acoplanarity was $40^{\circ}$. Pairs with smaller missing energy had to have transverse momentum above $25 \mathrm{GeV} / \mathrm{c}$ and acoplanarity above $40^{\circ}$.

Finally, $\mathrm{W}^{+} \mathrm{W}^{-}$events were rejected by requiring the momentum of the more energetic of the two particles to be outside the range from $33 \mathrm{GeV} / c$ to $47 \mathrm{GeV} / c$ for the data taken at $161 \mathrm{GeV}$, and outside the range from $40 \mathrm{GeV} / c$ to $60 \mathrm{GeV} / c$ for the data taken at $172 \mathrm{GeV}$.

\subsection{Neutralino decays giving single $\gamma$ final states}

Anomalous production of single-photon events in DELPHI has been searched for at centre-of-mass energies $\sqrt{s} \approx 91 \mathrm{GeV}, 130 \mathrm{GeV}, 136 \mathrm{GeV}, 161 \mathrm{GeV}$, and $172 \mathrm{GeV}$. The data taken at the peak of the $Z$ resonance are included because of the large integrated luminosity collected at this energy. When interpreted in terms of $\tilde{\chi}_{1}^{0} \tilde{\chi}_{2}^{0}$ production with $\tilde{\chi}_{2}^{0} \rightarrow \tilde{\chi}_{1}^{0} \gamma$ these data improve the exclusion in the $\mu, M_{2}$ plane for low $\mu, M_{2}$, and $\tan \beta$ and improve the lower limits on neutralino and chargino masses.

The analyses of the data collected at $\sqrt{s} \approx 91 \mathrm{GeV}$ and at $130 \mathrm{GeV}$ and $136 \mathrm{GeV}$ are described in [22] and [4]. The analysis of the $161 \mathrm{GeV}$ and $172 \mathrm{GeV}$ data is similar to that of Ref. [4].

For all these centre-of-mass energies, the main Standard Model background is photons from initial state radiation in $\mathrm{e}^{+} \mathrm{e}^{-} \rightarrow \nu \bar{\nu} \gamma$ events As this background is strongly peaked in the forward region, only single-photon events with the photon detected in the barrel part of the electromagnetic calorimeter (HPC) were considered in the search for the $\mathrm{e}^{+} \mathrm{e}^{-} \rightarrow \tilde{\chi}_{1}^{0} \tilde{\mathrm{G}}$ process. The distribution of recoil mass in the selected single-photon events at $161 \mathrm{GeV}$ and $172 \mathrm{GeV}$ is shown in Fig. 2, together with that expected for the background.

\subsection{Neutralino decays giving $\gamma \gamma$ final states}

Events with two photons and missing energy could be a signature for the process $\mathrm{e}^{+} \mathrm{e}^{-} \rightarrow \tilde{\chi}_{1}^{0} \tilde{\chi}_{1}^{0}$ with both neutralinos decaying to $\gamma \tilde{\mathrm{G}}$. In this analysis the neutralino is assumed to be dominantly gaugino-like, and hence not to couple to the $Z$. In this case, if the gravitino is light, the photons will tend to be collinear for a light neutralino, while for large $M_{\tilde{\chi}_{1}^{0}}$ they will be acollinear and acoplanar. 
Photons reaching the calorimeters without converting were recognised as energy clusters in electromagnetic calorimeters not associated to charged particle tracks ('neutral clusters'). For efficient rejection of charged particles, no hits in the Vertex Detector aligned with calorimeter energy clusters were allowed. These criteria were complemented by the requirement that neutral clusters below $44^{\circ}$ or above $136^{\circ}$ in polar angle did not coincide in azimuth with TPC sector boundaries. Neutral clusters in the HPC not coinciding with module boundaries to within $1^{\circ}$, were required to have signals from three layers, each exceeding $5 \%$ of the shower energy. Associated energy deposits in the hadronic calorimeter were included, but were required not to exceed $15 \%$ of the electromagnetic cluster energy if not coincident with HPC module boundaries, otherwise the photon candidate was rejected.

Converted photons were recognised by the absence of hits in the Vertex Detector, and the requirement that the ratio between the energy deposited in the electromagnetic calorimeter and the momentum of charged particles be greater than 0.2 . There had to be eight or less reconstructed charged particle tracks associated to the energy cluster.

Events were selected if they had two such reconstructed photons in the polar angle range between $25^{\circ}$ and $155^{\circ}\left(30^{\circ}\right.$ and $150^{\circ}$ for converted photons) and no reconstructed charged particle tracks which were not associated with any of the photons. Any additional calorimetric clusters were required to have a total energy below $3 \mathrm{GeV}$. To reject cosmic ray events it was required that clusters in the hadron calorimeter above $3 \mathrm{GeV}$ had at least $90 \%$ of the energy detected in the first layer. Finally, events were eliminated if the missing momentum pointed in the direction of a $40^{\circ}$ tagger giving a signal.

A total of 141 events passed this preselection, while the expectation from the dominant background, the QED process $\mathrm{e}^{+} \mathrm{e}^{-} \rightarrow \gamma \gamma$, is $150 \pm 2$ events. Fig. 3 shows the distribution of acoplanarity versus energy for this data sample.

Following the preselection, additional criteria were imposed to eliminate the dominant QED background and enhance the signal. Two different sets of criteria were used, optimised for a light neutralino and a heavy neutralino, respectively.

The region of low neutralino mass was probed using photons detected in the HPC. It was required that the acollinearity between the two photons was below $20^{\circ}$, that the total energy in the event was less than $75 \%$ of the centre-of-mass energy, and that neither photon was within $1^{\circ}$ of an HPC module boundary. The last condition serves to optimise the energy reconstruction.

For the high mass region, the acoplanarity had to exceed $3^{\circ}$, and the total energy was required to be less than $90 \%$ of the centre-of-mass energy. To eliminate events with photons from initial state radiation or with poorly reconstructed photons, the missing momentum was required to be less than $0.1 \sqrt{s} / \mathrm{c}$ if its polar angle was within $10^{\circ}$ of the beam axis.

\section{Results in the case of a stable neutralino}

\subsection{Efficiencies and selected events}

The total numbers of background events expected in the different topologies are shown in tables 1 and 2, together with the numbers of events selected in the data at $\sqrt{s}=161 \mathrm{GeV}$ and $172 \mathrm{GeV}$.

The efficiencies of the chargino selections in sections 4.1 to 4.3 were estimated using 22 combinations of $\tilde{\chi}_{1}^{ \pm}$and $\tilde{\chi}_{1}^{0}$ masses in three chargino mass ranges $\left(M_{\tilde{\chi}_{1}^{ \pm}} \approx 80 \mathrm{GeV} / c^{2}\right.$, 


\begin{tabular}{|c|c|c|c|c|c|c|}
\hline & \multicolumn{6}{|c|}{ Chargino channels } \\
\hline & \multicolumn{3}{|c|}{ Non-degenerate selection } & \multicolumn{3}{|c|}{ Degenerate selection } \\
\hline Section: & 4.1 & 4.2 & 4.3 & 4.1 & 4.2 & 4.3 \\
\hline Topology: & $j j \ell$ & jets & $\ell \ell$ & $j j \ell$ & jets & $\ell \ell$ \\
\hline & \multicolumn{6}{|c|}{$161 \mathrm{GeV}$} \\
\hline Obs. events: & 0 & 2 & 0 & 0 & 0 & 0 \\
\hline Background: & $0.03 \pm 0.01$ & $1.8 \pm 0.3$ & $0.39 \pm 0.06$ & $0.47 \pm 0.07$ & $0.53 \pm 0.08$ & $0.51 \pm 0.08$ \\
\hline & \multicolumn{6}{|c|}{$172 \mathrm{GeV}$} \\
\hline Obs. events: & 0 & 0 & 1 & 0 & 1 & 0 \\
\hline Background: & $0.20 \pm 0.05$ & $1.4 \pm 0.2$ & $0.8 \pm 0.2$ & $0.28 \pm 0.04$ & $0.27 \pm 0.05$ & $0.32 \pm 0.07$ \\
\hline
\end{tabular}

Table 1: The number of events observed and the expected number of background events in the different chargino search channels under the hypothesis of a stable neutralino (sections 4.1 to 4.3 ). For $172 \mathrm{GeV}$, in the low $|\mu|$ and $M_{2}$ region where $\tilde{\chi}_{1}^{ \pm} \rightarrow \tilde{\chi}_{2}^{0} \mathrm{f} \overline{\mathrm{f}} \rightarrow \tilde{\chi}_{1}^{0} \mathrm{f} \overline{\mathrm{f}} \gamma$ decays were important, events with one or two isolated photons were also included. This led to an additional expected background of $0.41 \pm 0.12$ events which is not included in this Table.

\begin{tabular}{||c||c|c||}
\hline \hline \multicolumn{1}{||c||}{} & \multicolumn{2}{c||}{ Neutralino channels } \\
Section: & 4.5 & 4.6 \\
Topology: & $j j$ & $\ell \ell$ \\
\hline \multicolumn{1}{|c||}{} & \multicolumn{2}{|c||}{$161 \mathrm{GeV}$} \\
Obs. events: & 2 & 1 \\
Background: & $2.6 \pm 0.2$ & $1.7 \pm 0.7$ \\
\hline Obs. events: & \multicolumn{2}{|c||}{$172 \mathrm{GeV}$} \\
Background: & $4.2 \pm 0.3$ & $1.9 \pm 0.3$ \\
\hline \hline
\end{tabular}

Table 2: The number of events observed and the expected number of background events in the different neutralino search channels under the hypothesis of a stable neutralino (sections 4.5 and 4.6). The two events selected in the $j j$ topology at $161 \mathrm{GeV}$ are the same as in the non-degenerate selection of the chargino jets topology. The background is larger than for the chargino analysis (table 1) because of the larger range of mass differences covered. It is dominated by $\mathrm{q} \overline{\mathrm{q}}(\gamma), \mathrm{W}^{+} \mathrm{W}^{-}$, and $W e \nu_{e}$ events.

$M_{\tilde{\chi}_{1}^{ \pm}} \approx 70 \mathrm{GeV} / c^{2}$, and $\left.M_{\tilde{\chi}_{1}^{ \pm}} \approx 60 \mathrm{GeV} / c^{2}\right)$ and with $\Delta M$ ranging from $3 \mathrm{GeV} / c^{2}$ to $77 \mathrm{GeV} / c^{2}$. A total of 86000 chargino events was generated and passed through the complete simulation of the DELPHI detector.

In addition, 126000 such events were simulated for 63 different parameter combinations in the region of low $|\mu|$ and $M_{2}$, for which a special study was carried out (see section 5.2). In this region, the decay $\tilde{\chi}_{1}^{ \pm} \rightarrow \tilde{\chi}_{2}^{0} \mathrm{f} \bar{f} \rightarrow \tilde{\chi}_{1}^{0} f \bar{f} \gamma$ is important, and events with one or two isolated photons were therefore also included. Such events were selected by the analysis of section 4.4, leading to an increase of the efficiency for large $\Delta M$.

Fig. 4 shows the detection efficiencies at $172 \mathrm{GeV}$ for the three channels, considering for each channel all the searches in sections 4.1 to 4.4 . The jets search gives a large contribution to the efficiency for the $j j \ell$ channel, increasing the efficiency for it by $\sim 30 \%$ [5]. The contribution of the $j j \ell$ channel to the efficiency of the jets and $\ell$ searches is small. The overall detection efficiencies are shown in Fig. $4 \mathrm{~d}$. 
For the neutralino analysis, a total of $120000 \tilde{\chi}_{1}^{0} \tilde{\chi}_{2}^{0}$ events was generated for 39 different combinations of $M_{\tilde{\chi}_{2}^{0}}$ and $M_{\tilde{\chi}_{1}^{0}}$ masses with $M_{\tilde{\chi}_{2}^{0}}$ ranging from $23 \mathrm{GeV} / c^{2}$ to $150 \mathrm{GeV} / c^{2}$, and for different $\tilde{\chi}_{2}^{0}$ decay modes $\left(q \bar{q} \tilde{\chi}_{1}^{0}, \mu^{+} \mu^{-} \tilde{\chi}_{1}^{0}, \mathrm{e}^{+} \mathrm{e}^{-} \tilde{\chi}_{1}^{0}\right)$. The efficiencies for the neutralino selections in sections 4.5 and 4.6 are shown in Fig. 5.

In the $161 \mathrm{GeV}$ data, two hadronic events and one event in the neutralino $\ell$ topology were selected. Both hadronic events satisfied both the chargino jets selection criteria for the non-degenerate case and the neutralino $j j$ criteria. One of these events is shown in Fig. 6. The best estimate for the invariant mass of the two-jet system in this event, corrected for particle losses, is $(72 \pm 11) \mathrm{GeV} / c^{2}$, with a recoil mass of $(59 \pm 19) \mathrm{GeV} / c^{2}$. The most probable explanation of this event is $t$-channel We $\nu$ production where the electron is lost. The expected number of such events selected is 0.6. In the second event selected, both jets point to the regions of incomplete calorimeter coverage at $\theta \approx$ $40^{\circ}$. Hence the missing transverse momentum is likely to be an instrumental effect. The transverse momentum of the third event, selected in the neutralino $\ell$ topology, is just above the minimum accepted by the selection, and it is most likely a two-photon interaction.

In the $172 \mathrm{GeV}$ data, three hadronic and two leptonic events were selected, consistent with the expected Standard Model background. One is a likely $\mathrm{W}^{+} \mathrm{W}^{-}$event, with one $W$ decaying hadronically and the other into $\tau \nu$; the other two hadronic events and one leptonic event have low transverse momenta and are most likely due to two-photon interactions. The remaining leptonic event has high visible mass and could be due to a $\mathrm{W}^{+} \mathrm{W}^{-}$pair with both bosons decaying leptonically.

\subsection{Limits}

Limits on chargino production

The simulated data points were used to parametrise the efficiencies of the chargino selection criteria described in sections 4.1 to 4.4 in terms of $\Delta M$ and the mass of the chargino. Then a large number of SUSY points were investigated and the values of $\Delta M$, the chargino and neutralino masses and the various decay branching ratios were determined for each point. By applying the appropriate efficiency and branching ratios for each channel, the number of expected signal events for a given cross-section can be calculated. Taking also the expected background and the number of events actually observed into account, the $95 \%$ confidence level upper limit on the number of observed signal events can be calculated [23] and certain cross sections, or the corresponding points in the MSSM parameter space $\left(\mu, M_{2}, \tan \beta\right)$, may be excluded.

Figs. 7 and 8 show the chargino production cross-sections as obtained in the MSSM at $\sqrt{s}=172 \mathrm{GeV}$ for different chargino masses for the non-degenerate and degenerate cases. The parameters $M_{2}$ and $\mu$ were varied randomly in the ranges $0 \mathrm{GeV} / c^{2}<M_{2}<$ $800 \mathrm{GeV} / c^{2}$ and $-400 \mathrm{GeV} / c^{2}<\mu<400 \mathrm{GeV} / c^{2}$ for three different values of $\tan \beta$, namely $1,1.5$ and 35 . Since the expected cross-section depends strongly on the sneutrino mass, two different cases were considered: $41 \mathrm{GeV} / c^{2}<M_{\tilde{\nu}}<100 \mathrm{GeV} / c^{2}$ (light sneutrino) and $M_{\tilde{\nu}}>300 \mathrm{GeV} / c^{2}$ (heavy sneutrino). Applying the appropriate efficiency for each of the points shown the minimum non-excluded $M_{\tilde{\chi}_{1}^{ \pm}}$was determined.

As already mentioned, a special study was made of the region of small $|\mu|, M_{2}$ and $\tan \beta$ in order to derive mass limits for $M_{\tilde{\chi}_{1}^{0}}$, in particular. Whenever $\Delta M$ is large, the cascade decay $\tilde{\chi}_{1}^{ \pm} \rightarrow \tilde{\chi}_{2}^{0} \mathrm{f} \bar{f} \rightarrow \tilde{\chi}_{1}^{0} \gamma \mathrm{ff}$ becomes important, giving an energetic photon. The analysis described in section 4.4 , developed for the case of an unstable $\tilde{\chi}_{1}^{0}$ decaying to $\tilde{\mathrm{G}} \gamma$, then complements the analyses of sections 4.1 to 4.3 , which have low efficiencies for 
large $\Delta M$. However, close to $\mu=M_{2}=0$, both analyses fail since $\Delta M$ is large while the neutralino mass difference, and hence the photon energy, is small. In this case the chargino events would resemble $W^{+} W^{-}$events and, from the observed rate of $W^{+} W^{-}$ events in DELPHI [24], an absolute upper limit at 95\% confidence level on the chargino production cross-section of $3.1 \mathrm{pb}$ was derived for those points not otherwise excluded. Since the predicted chargino cross-section in all these points is above $3.5 \mathrm{pb}$, the entire region of small $|\mu|$ and $M_{2}$ can be excluded for a heavy sneutrino.

To derive the chargino mass limits, constraints on the process $Z \rightarrow \tilde{\chi}_{1}^{0} \tilde{\chi}_{2}^{0} \rightarrow \tilde{\chi}_{1}^{0} \tilde{\chi}_{1}^{0} \gamma$ were also included. These were derived from the DELPHI results on single-photon production at LEP 1 [22] mentioned in section 4.7 and discussed below in section 6.1 . For each $\left(\mu, M_{2}\right)$ combination the number of single-photon events compatible with $M_{\tilde{\chi}_{1}^{0}}$ and $M_{\tilde{\chi}_{2}^{0}}$, the number expected from the Standard Model background, and the energy-dependent efficiency were used in order to establish an upper limit on the cross-section times branching ratio, excluding certain MSSM parameter combinations.

The chargino mass limits are summarized in Table 3. The table also gives, for each case, the minimum MSSM cross-section excluded by the requirement that $M_{\tilde{\chi}_{1}^{ \pm}}$be above the appropriate limit. These cross-section values are also displayed in Figs. 7 and 8 . The excluded region in the plane of neutralino mass versus chargino mass, assuming a heavy sneutrino, is shown in Fig.9.

\section{Chargino mass limits in the non-degenerate case}

In the non-degenerate case with a large sneutrino mass $\left(>300 \mathrm{GeV} / c^{2}\right)$, the lower limit for the chargino using only data taken at $\sqrt{s}=172 \mathrm{GeV}$ ranges between $84.3 \mathrm{GeV} / c^{2}$ (for a mostly higgsino-like chargino) and the kinematic limit (for a mostly wino-like chargino). This limit, shown in Fig. 7, is beyond the kinematic limit for the $161 \mathrm{GeV}$ data.

For low sneutrino mass, a combination of data from different energies, taking into account the different efficiencies and the different dependence of the cross-section on the chargino and on the sneutrino masses, gives a better limit than $172 \mathrm{GeV}$ data alone. Using the number of expected signal and background events in the combined data from $130 \mathrm{GeV}$ to $172 \mathrm{GeV}$ together with the experimental upper bound of 4.96 events, the lower limit on the chargino mass of $67.6 \mathrm{GeV} / c^{2}$ shown in Fig. 7 was derived.

Chargino mass limits in the degenerate case

In the degenerate case $\left(\Delta M=5 \mathrm{GeV} / c^{2}\right)$, the cross-section does not depend significantly on the sneutrino mass, since the chargino is higgsino-like under the assumption of gaugino mass unification. The lower limit for the chargino mass, shown in Fig. 8, is $80.3 \mathrm{GeV} / c^{2}$. In the extremely degenerate case of $\Delta M=3 \mathrm{GeV} / c^{2}$, the corresponding mass limit is $52.4 \mathrm{GeV} / c^{2}$.

\section{Limits on neutralino production}

Limits on neutralino production in the case of a stable $\tilde{\chi}_{1}^{0}$ were derived from the parametrised efficiencies of sections 4.5 and 4.6 and the observed number of events, in the same way as for charginos. The limits obtained for the $\tilde{\chi}_{1}^{0} \tilde{\chi}_{2}^{0}$ production cross-section are shown in Figs. 10a, $\mathrm{b}$ and $\mathrm{c}$ assuming leptonic or hadronic decay modes. The limit obtained assuming that the $\tilde{\chi}_{2}^{0} \rightarrow \tilde{\chi}_{1}^{0} f \bar{f}$ decay is mediated by a $Z^{*}$, including both leptonic and hadronic modes and $20 \%$ of invisible final states, is presented in Fig. 10d. These limits also apply to $\tilde{\chi}_{1}^{0} \tilde{\chi}_{k}^{0}$ production with $\tilde{\chi}_{k}^{0}$ decaying into $\tilde{\chi}_{1}^{0}$.

\section{Limits on MSSM parameters and neutralino mass}

The result of the searches for charginos and neutralinos in the different topologies for a stable neutralino can be interpreted in the MSSM with a universal GUT scale gaugino 


\begin{tabular}{|c|c|c|c|c|}
\hline \multirow[t]{2}{*}{ Scenario } & \multicolumn{2}{|c|}{ Light sneutrino } & \multicolumn{2}{|c|}{ Heavy sneutrino } \\
\hline & $\begin{array}{c}M_{\chi^{ \pm}}^{\min } \\
\left(\mathrm{GeV} / c^{2}\right)\end{array}$ & $\begin{array}{l}\sigma^{\max } \\
(\mathrm{pb})\end{array}$ & $\begin{array}{c}M_{\chi^{ \pm}}^{\min } \\
\left(\mathrm{GeV} / c^{2}\right)\end{array}$ & $\begin{array}{l}\sigma^{\max } \\
(\mathrm{pb})\end{array}$ \\
\hline \multicolumn{5}{|c|}{ Stable neutralino } \\
\hline$\Delta M>10 \mathrm{GeV} / c^{2}$ & 67.6 & 0.65 & 84.3 & 0.82 \\
\hline$\Delta M=5 \mathrm{GeV} / c^{2}$ & 80.3 & 1.84 & 80.3 & 1.84 \\
\hline \multicolumn{5}{|c|}{ Unstable neutralino } \\
\hline$\Delta M>10 \mathrm{GeV} / c^{2}$ & 71.8 & 0.44 & 84.5 & 0.75 \\
\hline$\Delta M=1 \mathrm{GeV} / c^{2}$ & 84.5 & 0.75 & 84.5 & 0.75 \\
\hline
\end{tabular}

Table 3: 95\% confidence level limits for the chargino mass and the corresponding pair production cross-sections at $172 \mathrm{GeV}$ for the non-degenerate and a highly degenerate scenarios. The cases of a stable $\tilde{\chi}_{1}^{0}$ and $\tilde{\chi}_{1}^{0} \rightarrow \tilde{\mathrm{G}}_{\gamma}$, as well as a light $\left(41 \mathrm{GeV} / c^{2}<m_{\tilde{\nu}}<100 \mathrm{GeV} / c^{2}\right)$ and a heavy $\left(m_{\tilde{\nu}}>300 \mathrm{GeV} / c^{2}\right)$ sneutrino, are considered.

mass parameter. This yields the exclusion regions in the $\left(\mu, M_{2}\right)$ plane shown in Fig. 11 for different values of $\tan \beta$, assuming a heavy sneutrino and a heavy selectron $\left(m_{0}=\right.$ $\left.1 \mathrm{TeV} / \mathrm{c}^{2}\right)$. When different event selections contributed to the same physical production channel, the efficiency and background of a 'logical or' of the channels was used; otherwise, when they contributed to different channels, the method of Ref. [25] was used to combine the selections. These limits, based on data taken at $\sqrt{s}=161 \mathrm{GeV}$ and $172 \mathrm{GeV}$, improve on previous limits from $\sqrt{s}=130-136 \mathrm{GeV}$, and represent a significant increase in range as compared to LEP 1 results [26]. The neutralino analysis independently excludes a substantial part of the region covered by the chargino search, and marginally extends this region for low $\tan \beta$.

Note that the neutralino masses, and hence the regions in the $\left(\mu, M_{2}\right)$ plane excluded by the neutralino search, depend directly on the assumed GUT relation $M_{1} / M_{2}=\frac{5}{3} \tan ^{2} \theta_{W} \approx$ 0.5. The region excluded by the chargino search, on the other hand, depends on this ratio only via the effect of decay kinematics on the efficiency. While the $M_{\tilde{\chi}_{1}^{0}}+M_{\tilde{\chi}_{2}^{0}}$ isomass contours relevant to the present neutralino search depend only weakly on this ratio, any consideration about the range of $M_{\tilde{\chi}_{1}^{0}}$ that can be excluded depends sensitively on the relation between $M_{1}$ and $M_{2}$.

Under the assumption that $M_{1} / M_{2} \gtrsim 0.5$, the excluded regions in the $\left(\mu, M_{2}\right)$ plane can be translated into a limit on the mass of the lightest neutralino. This limit depends on $\tan \beta$, and for $\tan \beta$ close to one it may be improved by including the DELPHI results [22] on the process $\mathrm{Z} \rightarrow \tilde{\chi}_{1}^{0} \tilde{\chi}_{2}^{0} \rightarrow \tilde{\chi}_{1}^{0} \tilde{\chi}_{1}^{0} \gamma$ (see above and section 6.1 ). The region excluded by these results for $\tan \beta=1$ and large $m_{0}$ is shown in Fig. 12, together with the regions excluded by the higher energy data and the $M_{\tilde{\chi}_{1}^{0}}=24.9 \mathrm{GeV} / c^{2}$ contour corresponding to the limit on $M_{\tilde{\chi}_{1}^{0}}$ for this $\tan \beta$. Fig. 13 gives the limit on $M_{\tilde{\chi}_{1}^{0}}$ as a function of $\tan \beta$. 


\section{Results in the case of an unstable neutralino}

\subsection{Efficiencies and selected events}

Chargino $\gamma \gamma X$ topologies

The efficiency of the chargino selection for an unstable neutralino decaying into a photon and a gravitino was calculated from a total of 15000 events generated using the same combinations of $M_{\tilde{\chi}_{1}^{ \pm}}$and $M_{\tilde{\chi}_{1}^{0}}$ as above. As mentioned in section 4.4, the same selection applies to all topologies. The efficiency, as shown in Fig. 14, varies only weakly with $\Delta M$ and is around $50 \%$. Note that, due to the presence of the photons from the neutralino decay, the region of high degeneracy (down to $\Delta M=1 \mathrm{GeV} / c^{2}$ ) is fully covered. No events were found at any of the centre-of-mass energies (130-136 GeV, $161 \mathrm{GeV}$, and $172 \mathrm{GeV}$ ). The total expected background is $1.5 \pm 0.4$ events.

Neutralino single- $\gamma$ topology

At $\sqrt{s} \approx 91 \mathrm{GeV}[22]$, the analysis in the barrel region of the DELPHI detector $\left(\left|\cos \theta_{\gamma}\right|<0.7\right.$, where $\theta_{\gamma}$ is the photon polar angle) yielded 11 events with $E_{\gamma}>10 \mathrm{GeV}$, four of which had $E_{\gamma}>15 \mathrm{GeV}$. No events with photon energy greater than $22 \mathrm{GeV}$ were found in the data. The expected number of events from the reaction $\mathrm{e}^{+} \mathrm{e}^{-} \rightarrow \nu \bar{\nu} \gamma$ in the case of three neutrino generations is $8.2 \pm 0.6$ and $2.9 \pm 0.2$ for photon energies above $10 \mathrm{GeV}$ and $15 \mathrm{GeV}$, respectively. The contamination from $\mathrm{e}^{+} \mathrm{e}^{-} \rightarrow \mathrm{e}^{+} \mathrm{e}^{-} \gamma$ events with the final state electrons escaping detection is negligible.

At $\sqrt{s}=130 \mathrm{GeV}-136 \mathrm{GeV}$ [4], the search for single-photon events yielded 19 events with $E_{\gamma}>4 \mathrm{GeV}$ in the barrel region, in agreement with the standard model expectation of $18.5 \pm 0.5$ events. Finally, an analysis similar to [4] yielded 11 events at $\sqrt{s}=161 \mathrm{GeV}$ and 11 events at $172 \mathrm{GeV}$, while $15.6 \pm 0.4$ and $10.8 \pm 0.4$ were predicted from Standard Model sources at these two energies. In summary, the searches for $\tilde{\chi}_{1}^{0} \tilde{G}$ production gave no significant excess of single-photon events as shown in table 4.

The signal efficiency was found to be between $30 \%$ and $48 \%$ depending on $\sqrt{s}$ and $M_{\tilde{\chi}_{1}^{0}}$, taking into account the trigger efficiency. The trigger efficiency for the sample of photons energy above $20 \mathrm{GeV}$ used in the analysis was $80 \%$ for $161 \mathrm{GeV}$ data taking period and $68 \%$ for $172 \mathrm{GeV}$ data taking period.

\begin{tabular}{||c||c|c|c|c||}
\hline \hline \multicolumn{1}{||c||}{} & \multicolumn{4}{|c||}{ Unstable neutralino single- $\gamma$ topology } \\
\hline$\sqrt{s}$ & $91 \mathrm{GeV}$ & $130-136 \mathrm{GeV}$ & $161 \mathrm{GeV}$ & $172 \mathrm{GeV}$ \\
Minimum $E_{\gamma}$ & $10 \mathrm{GeV}$ & $4 \mathrm{GeV}$ & $4 \mathrm{GeV}$ & $4 \mathrm{GeV}$ \\
Obs. events: & 11 & 19 & 11 & 11 \\
Background: & $8.2 \pm 0.6$ & $18.5 \pm 0.5$ & $15.6 \pm 0.4$ & $10.8 \pm 0.4$ \\
\hline \hline
\end{tabular}

Table 4: The number of events observed and the expected number of background events in the unstable neutralino single- $\gamma$ topology. As explained in the text (sections 4.7 and 6.1 ), different selection criteria were applied for the data taken at different centre-of-mass energies.

If the $\tilde{\chi}_{1}^{0}$ lifetime is long enough for the decay to take place far from the interaction region, the efficiency could be considerably reduced. This is because the shower axis reconstructed in the $\mathrm{HPC}$ was required to point to within $15^{\circ}$ of the beam interaction region in order to suppress noise and cosmic interactions. For the process $\mathrm{e}^{+} \mathrm{e}^{-} \rightarrow \tilde{\chi}_{1}^{0} \tilde{\mathrm{G}}_{\mathrm{o}}$ to occur with a significant cross-section, however, the gravitino has to be lighter than about $10^{-5} \mathrm{eV} / \mathrm{c}^{2}[27]$, and in this case the neutralino lifetime is negligibly short. 
Neutralino $\gamma \gamma$ topology

The efficiency for the process $\mathrm{e}^{+} \mathrm{e}^{-} \rightarrow \tilde{\chi}_{1}^{0} \tilde{\chi}_{1}^{0}$, with $\tilde{\chi}_{1}^{0} \rightarrow \tilde{\mathrm{G}} \gamma$ (section 4.8), depends on the $\tilde{\chi}_{1}^{0}$ mass and ranges from $32 \%$ for a light neutralino, to $49 \%$ for the heavy neutralino search, including the trigger efficiency. The trigger efficiency depended on the data taking period and was $96 \%$ for $161 \mathrm{GeV}$ data and $75 \%$ for $172 \mathrm{GeV}$ data. Contrary to the single-photon case, the efficiency for the $\mathrm{e}^{+} \mathrm{e}^{-} \rightarrow \tilde{\chi}_{1}^{0} \tilde{\chi}_{1}^{0}$ channel is essentially independent of the neutralino lifetime, provided that the $\tilde{\chi}_{1}^{0} \rightarrow \tilde{\mathrm{G}} \gamma$ decay occurs within a distance of $\sim 1 \mathrm{~m}$ from the beam interaction point. No event passed the selection criteria for a light neutralino, while two events in the $172 \mathrm{GeV}$ data were selected by the heavy neutralino search. These are the two events at high acoplanarity in Fig. 3. The expected number of events from $\nu \bar{\nu} \gamma \gamma(\gamma)$, including a possibility of a third photon from initial state radiation collinear with the beam, is $2.2 \pm 0.2$. Fig. 15 shows the mass of the unseen recoil system as calculated for the two events, compared to the expected distribution obtained using the CompHEP [28] program. The calculation of Ref. [8], based on the same program, gives only a small probability of observing recoil masses significantly above the $\mathrm{Z}$ mass. As is shown in Fig. 16, however, including the possibility of a third photon from initial state radiation collinear with the beam, together with the differences in the event selection, significantly increases this probability.

\begin{tabular}{|r|c|c|c|c|c|c|}
\hline \multirow{2}{*}{$\sqrt{s}$} & \multicolumn{3}{|c|}{ Light $\tilde{\chi}_{1}^{0}$} & \multicolumn{3}{c|}{ Heavy $\tilde{\chi}_{1}^{0}$} \\
\cline { 2 - 7 } & Data & QED & $\nu \bar{\nu} \gamma \gamma$ & Data & QED & $\nu \bar{\nu} \gamma \gamma$ \\
\hline \hline $130-136$ & 0 & $0.53 \pm 0.11$ & $0.00_{-0.00}^{+0.03}$ & 0 & $0.09 \pm 0.04$ & $0.94 \pm 0.13$ \\
\hline 161 & 0 & $0.80 \pm 0.14$ & $0.00_{-0.00}^{+0.03}$ & 0 & $0.24 \pm 0.08$ & $0.66 \pm 0.14$ \\
\hline 172 & 0 & $0.34 \pm 0.10$ & $0.015 \pm 0.003$ & 2 & $0.19 \pm 0.08$ & $0.61 \pm 0.02$ \\
\hline
\end{tabular}

Table 5: The number of events observed and the expected number of background events from the QED $\mathrm{e}^{+} \mathrm{e}^{-} \rightarrow \gamma \gamma$ and $\nu \bar{\nu} \gamma \gamma$ reactions.

\subsection{Limits}

The chargino cross-section limits corresponding to the case where the neutralino is unstable and decays via $\tilde{\chi}_{1}^{0} \rightarrow \tilde{\mathrm{G}} \gamma$ are also shown in Figs. 7 and 8 , and in table 3 . In the non-degenerate case the chargino mass limits at $95 \%$ confidence level are $84.5 \mathrm{GeV} / \mathrm{c}^{2}$ and $71.8 \mathrm{GeV} / \mathrm{c}^{2}$ for a heavy and light sneutrino, respectively, while in the degenerate case $\left(\Delta M=1 \mathrm{GeV} / c^{2}\right)$ the limit is $84.5 \mathrm{GeV} / c^{2}$.

The upper limits at $95 \%$ confidence level on the cross-sections $\sigma\left(\mathrm{e}^{+} \mathrm{e}^{-} \rightarrow \tilde{\chi}_{1}^{0} \tilde{\mathrm{G}}\right)$ and $\sigma\left(\mathrm{e}^{+} \mathrm{e}^{-} \rightarrow \tilde{\chi}_{1}^{0} \tilde{\chi}_{1}^{0}\right)$ when $\tilde{\chi}_{1}^{0} \rightarrow \tilde{\mathrm{G}} \gamma$ are shown in Fig. 17 as a function of $M_{\tilde{\chi}_{1}^{0}}$ for different values of the centre-of-mass energy. The limits assume that the neutralino decays close to the beam spot and that the branching ratio $\mathrm{BR}\left(\tilde{\chi}_{1}^{0} \rightarrow \tilde{\mathrm{G}} \gamma\right)$ is $100 \%$. As already stated, the limit on $\sigma\left(\mathrm{e}^{+} \mathrm{e}^{-} \rightarrow \tilde{\chi}_{1}^{0} \tilde{\chi}_{1}^{0}\right)$ is also valid for finite $\tilde{\chi}_{1}^{0}$ lifetimes, provided that the decay occurs within a distance of $\sim 1 \mathrm{~m}$ from the beam spot.

\section{Summary}

Searches for charginos and neutralinos at $\sqrt{s}=161 \mathrm{GeV}$ and $172 \mathrm{GeV}$ allow the exclusion of a large domain of SUSY parameters. 
Assuming a difference in mass between chargino and neutralino, $\Delta M$, of $10 \mathrm{GeV} / c^{2}$ or more, and a sneutrino heavier than $300 \mathrm{GeV} / \mathrm{c}^{2}$, the existence of a chargino lighter than $84.3 \mathrm{GeV} / c^{2}$ can be excluded. If a gaugino-dominated chargino is assumed in addition, the kinematical limit of $86 \mathrm{GeV} / c^{2}$ is reached. If $\Delta M$ is between $5 \mathrm{GeV} / c^{2}$ and $10 \mathrm{GeV} / c^{2}$, the lower limit on the chargino mass becomes $80.3 \mathrm{GeV} / c^{2}$, independent of the sneutrino mass, while for $\Delta M=3 \mathrm{GeV} / c^{2}$ it falls to $52.4 \mathrm{GeV} / c^{2}$.

For a light sneutrino and $\Delta M^{\dagger}$ of $10 \mathrm{GeV} / c^{2}$ or more, the lower limit on the chargino mass is $67.6 \mathrm{GeV} / c^{2}$.

Limits on the cross-section for $\tilde{\chi}_{1}^{0} \tilde{\chi}_{2}^{0}$ production of about $1 \mathrm{pb}$ are obtained, and the excluded region in the $\left(\mu, M_{2}\right)$ plane is extended by the combined use of the neutralino and chargino searches. A special study of the low $|\mu|, M_{2}, \tan \beta$ region gives a limit on the mass of the lightest neutralino, valid in the case of large $m_{0}$, of $24.9 \mathrm{GeV} / c^{2}$ at $95 \%$ confidence level.

A specific search was performed assuming the decay of the lightest neutralino into photon and gravitino, leading to upper limits on the $\tilde{\chi}_{1}^{0} \tilde{\chi}_{1}^{0}$ and $\tilde{\chi}_{1}^{0} \tilde{G}$ production crosssections of about $1.5 \mathrm{pb}$ in this case. In the same scenario, a dedicated search for $\tilde{\chi}_{1}^{+} \tilde{\chi}_{1}^{-}$ production gives somewhat more stringent limits on cross-sections and masses than in the case of a stable $\tilde{\chi}_{1}^{0}$.

\section{Acknowledgements}

We are greatly indebted to our technical collaborators and to the funding agencies for their support in building and operating the DELPHI detector. We also want to thank the members of the CERN accelerator divisons for the continued excellent performance of the LEP collider in the energy range above the $\mathrm{Z}$ resonance.

\footnotetext{
${ }^{\dagger}$ If the sneutrino mass is between those of the lightest neutralino and the chargino, $\Delta M$ is the mass difference between chargino and sneutrino.
} 


\section{References}

[1] P. Fayet and S. Ferrara, Phys. Rep. 32 (1977) 249;

H.P. Nilles, Phys. Rep. 110 (1984) 1;

H.E. Haber and G.L. Kane, Phys. Rep. 117 (1985) 75.

[2] DELPHI Coll., P. Abreu et al., Phys. Lett. B382 (1996) 323.

[3] DELPHI Coll., P. Abreu et al., Phys. Lett. B387 (1996) 651.

[4] DELPHI Coll., P. Abreu et al., Phys. Lett. B380 (1996) 471.

[5] P.Rebecchi, Optimisation de l'herméticité du détecteur DELPHI pour la recherche de particules supersymétriques à LEPD, Ph.D. thesis, LAL 96-30 (May 1996), Université Paris XI Orsay, CERN THESIS-96-003.

[6] D. Dicus et al., Phys. Rev. D41 (1990) 2347;

D. Dicus et al., Phys. Rev. D43 (1991) 2951;

D. Dicus et al., Phys. Lett. B258 (1991) 231.

[7] S. Dimopoulos et al., Phys. Rev. Lett. 76 (1996) 3494;

S. Ambrosanio et al., Phys. Rev. Lett. 76 (1996) 3498;

J.L. Lopez and D.V. Nanopoulos, Mod. Phys. Lett. A10 (1996) 2473;

J.L. Lopez and D.V. Nanopoulos, Phys. Rev. D55 (1997) 4450

[8] S. Ambrosanio et al., Phys. Rev. D54 (1996) 5395.

[9] A.Bartl, H.Fraas and W. Majerotto, Z. Phys. C30 (1986) 441;

A.Bartl, H.Fraas and W. Majerotto, Z. Phys. C41 (1988) 475;

A.Bartl, H.Fraas, W. Majerotto and B.Mösslacher, Z. Phys. C55 (1992) 257.

[10] DELPHI Coll., P. Aarnio et al., Nucl. Instr. and Meth. 303 (1991) 233;

DELPHI Coll., P. Abreu et al., Nucl. Instr. and Meth. 378 (1996) 57.

[11] S. Navas Concha, Búsqueda de charginos con el detector DELPHI de LEP a $E_{c m}=130-172 \mathrm{GeV}$, Ph.D. thesis, CERN-THESIS-97-005 (June 1997).

[12] T. Sjöstrand, Comp. Phys. Comm. 39 (1986) 347;

T. Sjöstrand, PYTHIA 5.6 and JETSET 7.3, CERN-TH/6488-92.

[13] DELPHI Coll., P. Abreu et al., Z. Phys. C73 (1996) 11.

[14] S. Katsanevas and S. Melachroinos in Physics at LEPD, CERN 96-01, Vol. 2, p. 328.

[15] S. Ambrosanio and B. Mele, Phys. Rev. D52 (1995) 3900;

S. Ambrosanio and B. Mele, Phys. Rev. D53 (1996) 2451.

[16] J.E. Campagne and R. Zitoun, Z. Phys. C43 (1989) 469.

[17] S. Jadach and Z. Was, Comp. Phys. Comm. 79 (1994) 503.

[18] F.A. Berends, R. Kleiss, W. Hollik, Nucl. Phys. B304 (1988) 712.

[19] F.A. Berends, R. Pittau, R. Kleiss, Comp. Phys. Comm. 85 (1995) 437.

[20] S. Nova, A. Olshevski, and T. Todorov, A Monte Carlo event generator for two photon physics, DELPHI note 90-35 (1990).

[21] F.A. Berends, P.H. Daverveldt, R. Kleiss, Comp. Phys. Comm. 40 (1986) 271, Comp. Phys. Comm. 40 (1986) 285, Comp. Phys. Comm. 40 (1986) 309.

[22] DELPHI Coll., P. Abreu et al., Z. Phys. C74 (1997) 577

[23] O. Helene, Nucl. Instr. and Meth. 212 (1983) 319.

[24] DELPHI Coll., P. Abreu et al., to appear.

[25] V.F. Obraztsov, Nucl. Instr. and Meth. 316 (1992) 388.

[26] ALEPH Coll., D. Decamp et al., Phys. Rep. 216 (1992) 253;

A. Lopez-Fernandez, DELPHI note 92-95 (Dallas) PHYS 206;

L3 Coll., M. Acciarri et al., Phys. Lett. B350 (1995) 109;

OPAL Coll., G. Alexander et al., Phys. Lett. B377 (1996) 273.

[27] J. Lopez, D. Nanopoulos and A. Zichichi, Phys. Rev. Lett. 77 (1996) 5168.

[28] E. Boos et al. in Physics at LEP2, CERN 96-01, Vol. 2, p. 16; hep-ph/9503280. 
LEPTONIC SELECTION
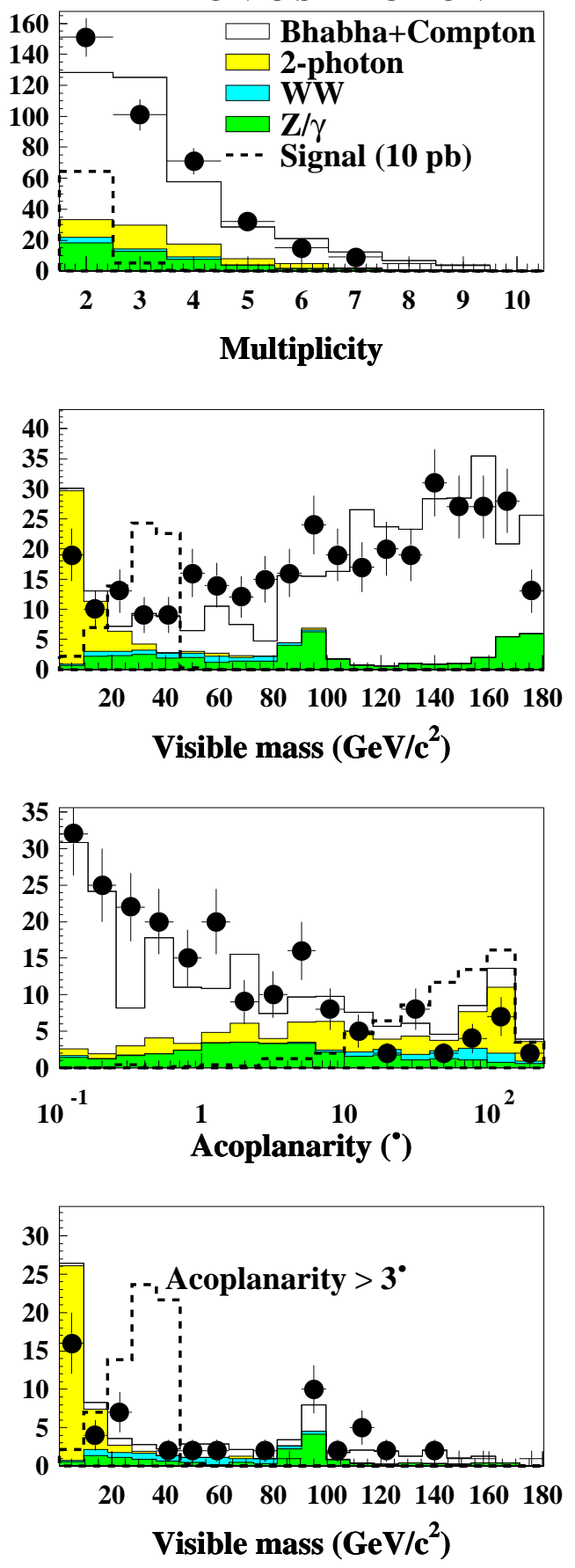
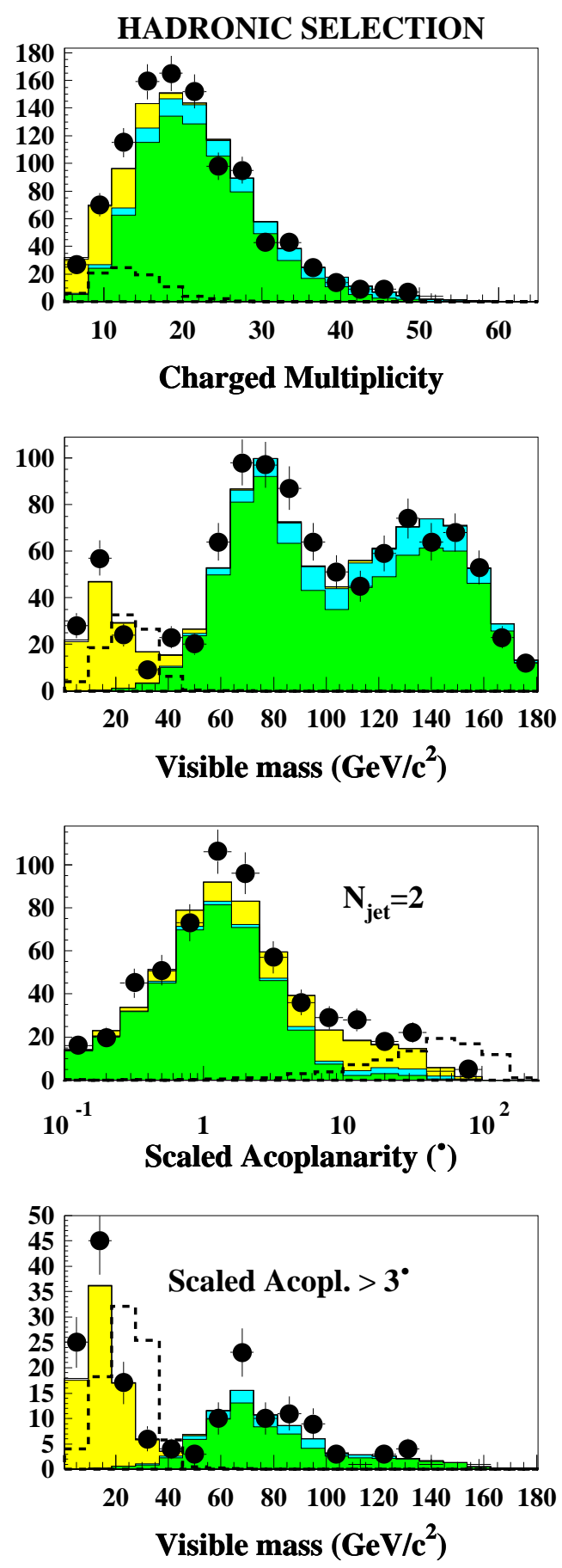

Figure 1: Distributions of multiplicities, visible mass, and acoplanarities for real data events (points) and simulated events (histograms) at $172 \mathrm{GeV}$, selected by typical 'leptonic' and 'hadronic' selections (see text). The scaled acoplanarity was calculated only for events where two jets were reconstructed using the LUCLUS algorithm with $d_{\text {join }}=$ $10 \mathrm{GeV} / c$. For the visible mass distributions at the bottom of the figure, acoplanar events were selected as indicated. The background is decomposed into its components (thin-bordered histograms with different shadings as shown in the first sub-figure). The fat-dashed unshaded histograms show the distributions expected for a $\tilde{\chi}_{1}^{0} \tilde{\chi}_{2}^{0}$ signal with $M_{\tilde{\chi}_{1}^{0}}=60 \mathrm{GeV} / c^{2}, M_{\tilde{\chi}_{2}^{0}}=105 \mathrm{GeV} / c^{2}$, and an arbitrarily chosen cross-section of $10 \mathrm{pb}$. 


\section{SINGLE PHOTON 161+172 GeV}

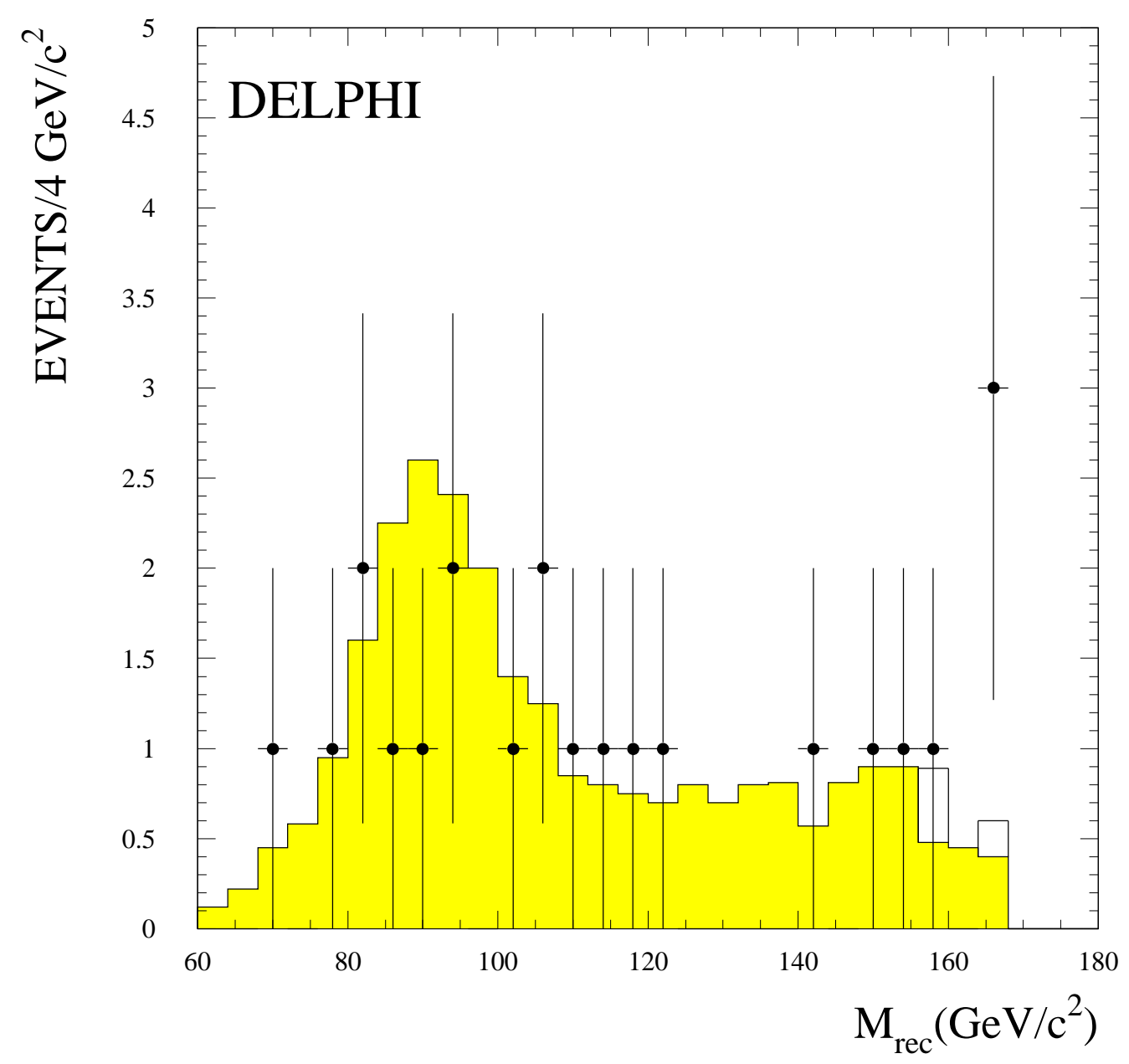

Figure 2: The distribution of recoil mass for the single-photon events selected in the data accumulated at $161 \mathrm{GeV}$ and $172 \mathrm{GeV}$ (points) and the expected background distribution (histogram). The shaded part of the histogram represents the $\mathrm{e}^{+} \mathrm{e}^{-} \rightarrow \nu \bar{\nu} \gamma$ background, while the unshaded part corresponds to $\mathrm{e}^{+} \mathrm{e}^{-} \rightarrow \mathrm{e}^{+} \mathrm{e}^{-} \gamma$ where the final state electrons are lost along the beam. 

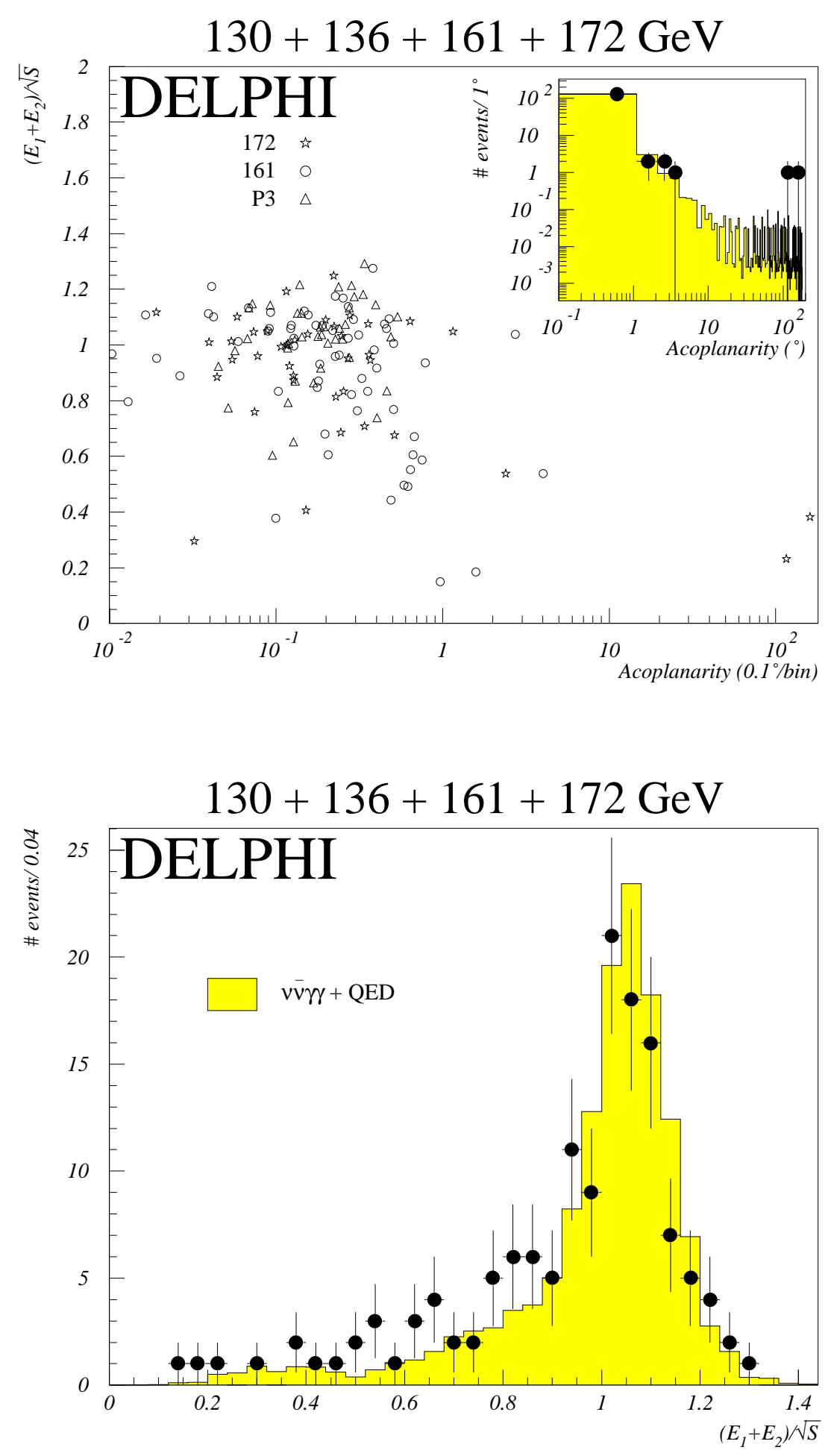

Figure 3: The distribution of acoplanarity and total energy normalised to the centre-of-mass energy for the selected events with two-photon final states for all energies. The data were collected in 1995 and 1996 at centre-of-mass energies between $130 \mathrm{GeV}$ and $172 \mathrm{GeV}$. The histograms show the sum of the expected backgrounds from $\gamma \gamma \nu \bar{\nu}$ and QED $\mathrm{e}^{+} \mathrm{e}^{-} \rightarrow \gamma \gamma$ events. 


\section{DELPHI $\tilde{\chi}_{1}^{+} \tilde{\chi}_{1}^{-}$efficiencies $(172 \mathrm{GeV})$}
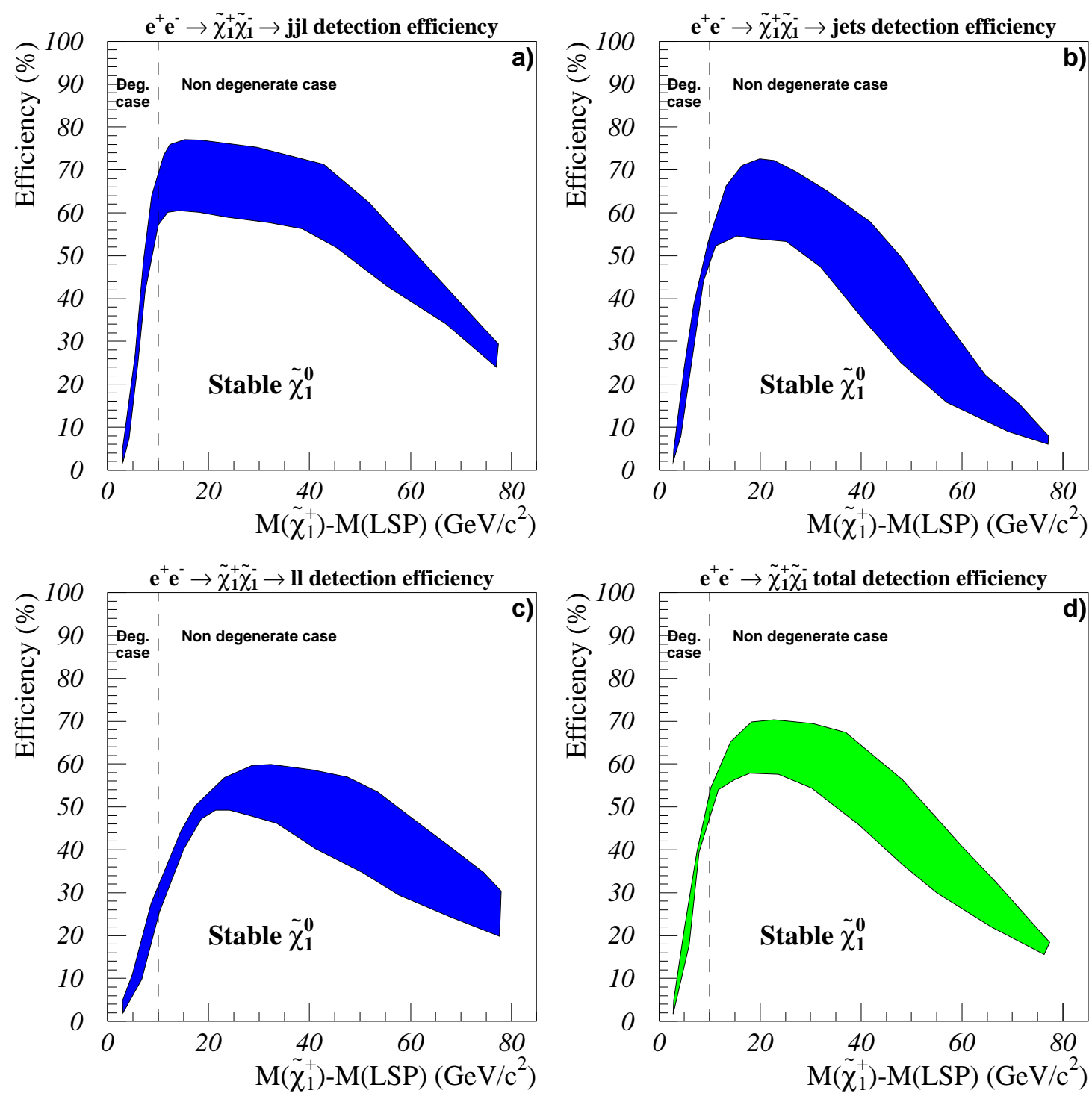

Figure 4: Chargino detection efficiencies for the three modes a) jjl, b) jets and c) $\ell \ell$ (including also photons from cascade decays via $\tilde{\chi}_{2}^{0}$ ), obtained by combining four sets of selection criteria (sections 4.1 to 4.4). d) Overall efficiency obtained by summing the three modes. The bands correspond to the statistical error in the simulation of about $2 \%$ absolute, combined with the effect of varying the assumed chargino mass between 60 and $80 \mathrm{GeV} / c^{2}$. 


\section{DELPHI $\tilde{\chi}_{1}^{0} \tilde{\chi}_{2}^{0}$ efficiencies}
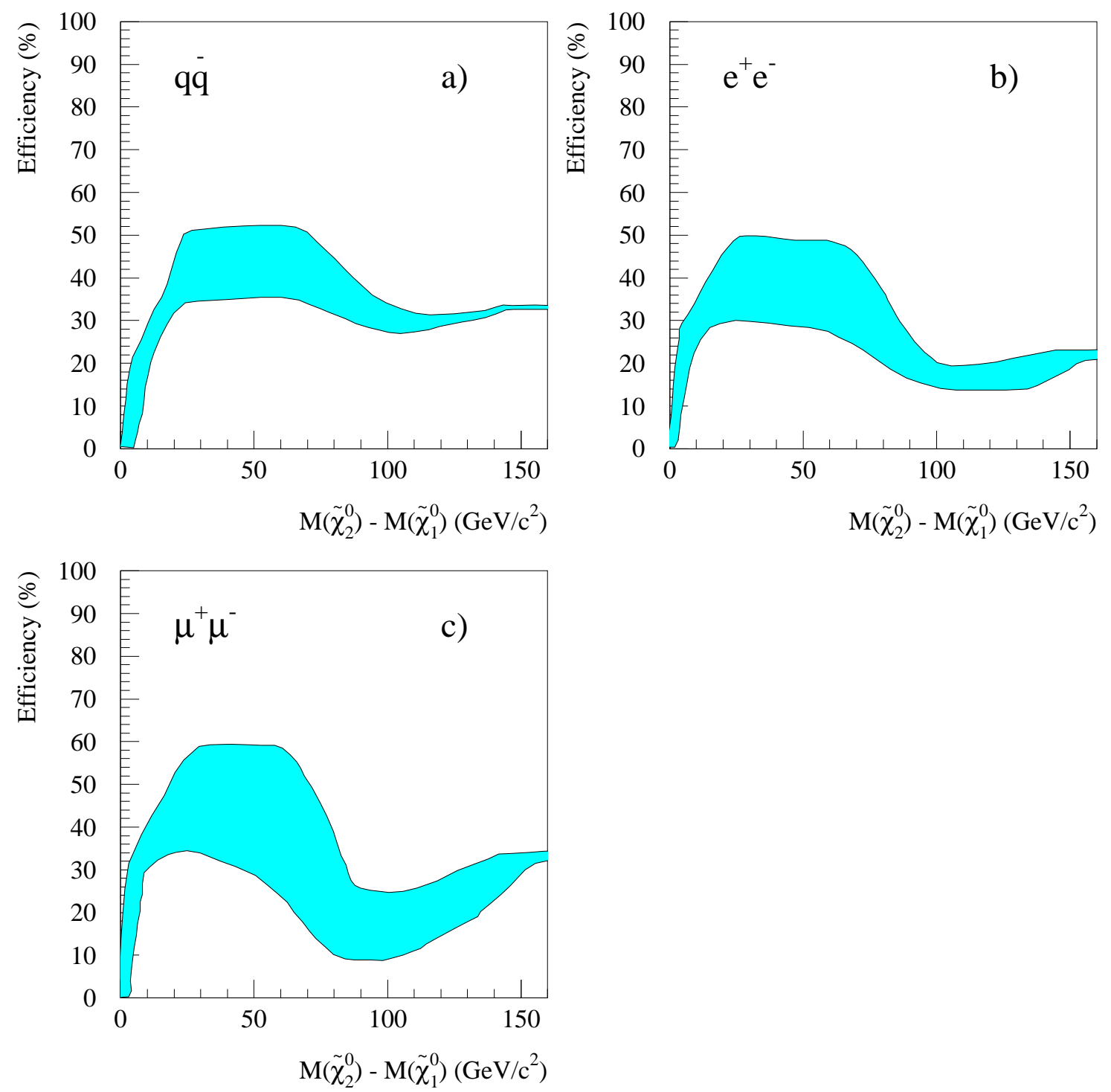

Figure 5: Neutralino pair production detection efficiency for the $j j$ and $\ell \ell$ topologies at $172 \mathrm{GeV}$ as functions of $M_{\tilde{\chi}_{2}^{0}}-M_{\tilde{\chi}_{1}^{0}}$. The efficiency drop around $90 \mathrm{GeV} / c^{2}$ is caused by the need to reject $Z(\gamma)$ events. The bands correspond to the statistical error in the simulation of about $2 \%$ absolute, combined with the effect of varying the $\tilde{\chi}_{2}^{0}$ mass between 23 and $150 \mathrm{GeV} / \mathrm{c}^{2}$. 


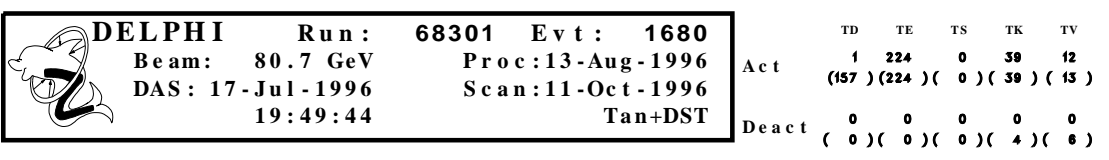

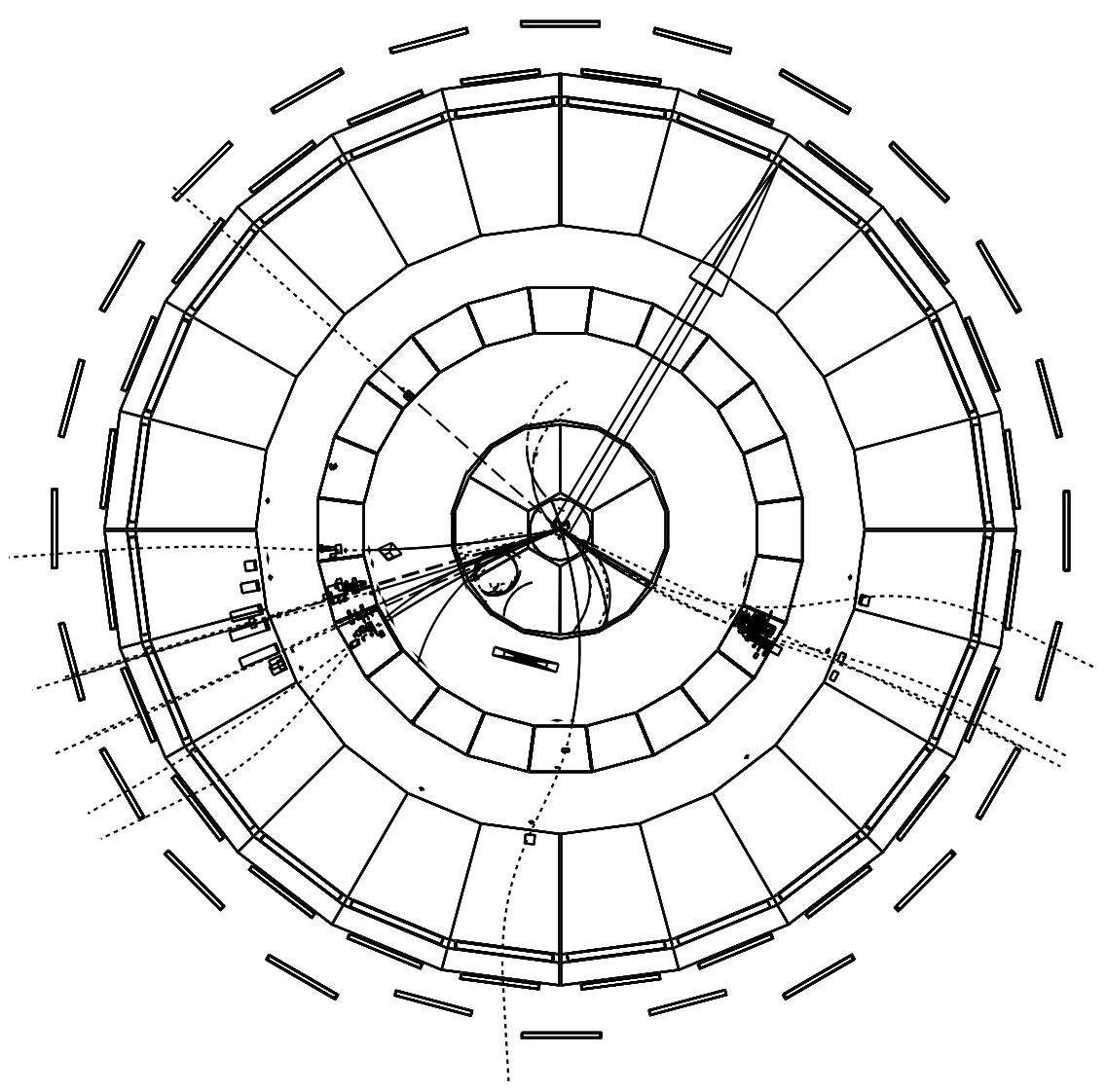

Figure 6: A selected chargino/neutralino candidate event in the jets and $j j$ topologies viewed in the projection perpendicular to the beam direction. The arrow indicates the missing momentum direction. The polar angle of the missing momentum is $81 \pm 1^{\circ}$. Charged particle trajectories are shown as solid curves in the regions of the tracking detectors and neutral particles as dashed lines. Both are shown as short dashes when extrapolated. Energy deposits in the electromagnetic and hadronic calorimeters are shown as boxes, and long dashes indicate such showers interpreted as neutral particles. 


\section{DELPHI $\tilde{\chi}_{1}^{+} \tilde{\chi}_{1}^{-}$limits at $172 \mathrm{GeV}$}

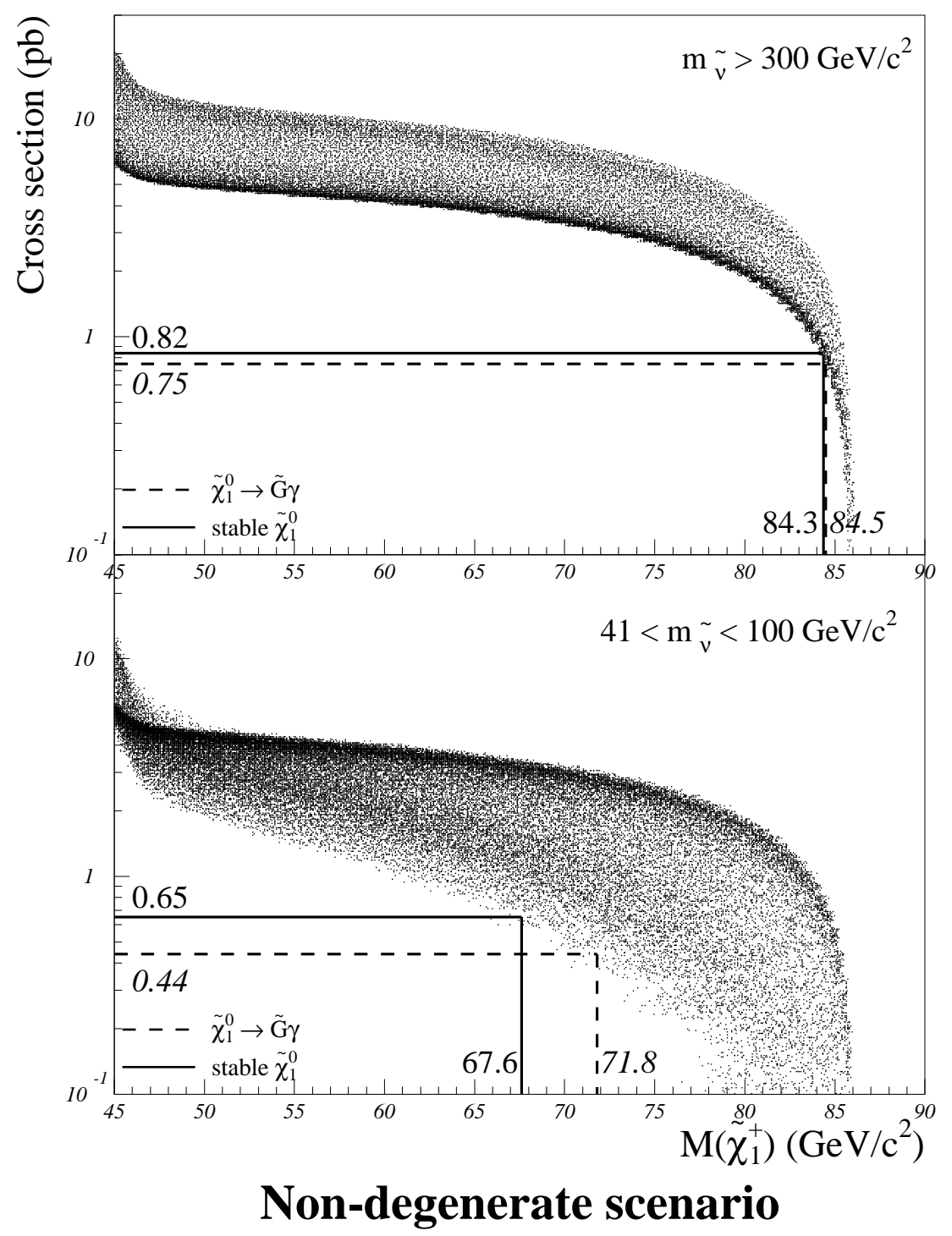

Figure 7: Expected cross-sections at $172 \mathrm{GeV}$ versus the chargino mass (dots) in the non-degenerate scenario $\left(\Delta M>10 \mathrm{GeV} / c^{2}\right)$ for different MSSM parameters. A heavy sneutrino $\left(m_{\tilde{\nu}}>300 \mathrm{GeV} / c^{2}\right)$ has been assumed in the upper plot and a light sneutrino (41 GeV/c $\left.c^{2}<m_{\tilde{\nu}}<100 \mathrm{GeV} / c^{2}\right)$ in the lower one. The cross-sections indicated are the minimum ones in the excluded mass region. The case of small $|\mu|$ and $M_{2}$ for $\tan \beta=1$ is discussed in detail in section 5.2. 


\section{DELPHI $\tilde{\chi}_{1}^{+} \tilde{\chi}_{1}^{-}$limits at $172 \mathrm{GeV}$}

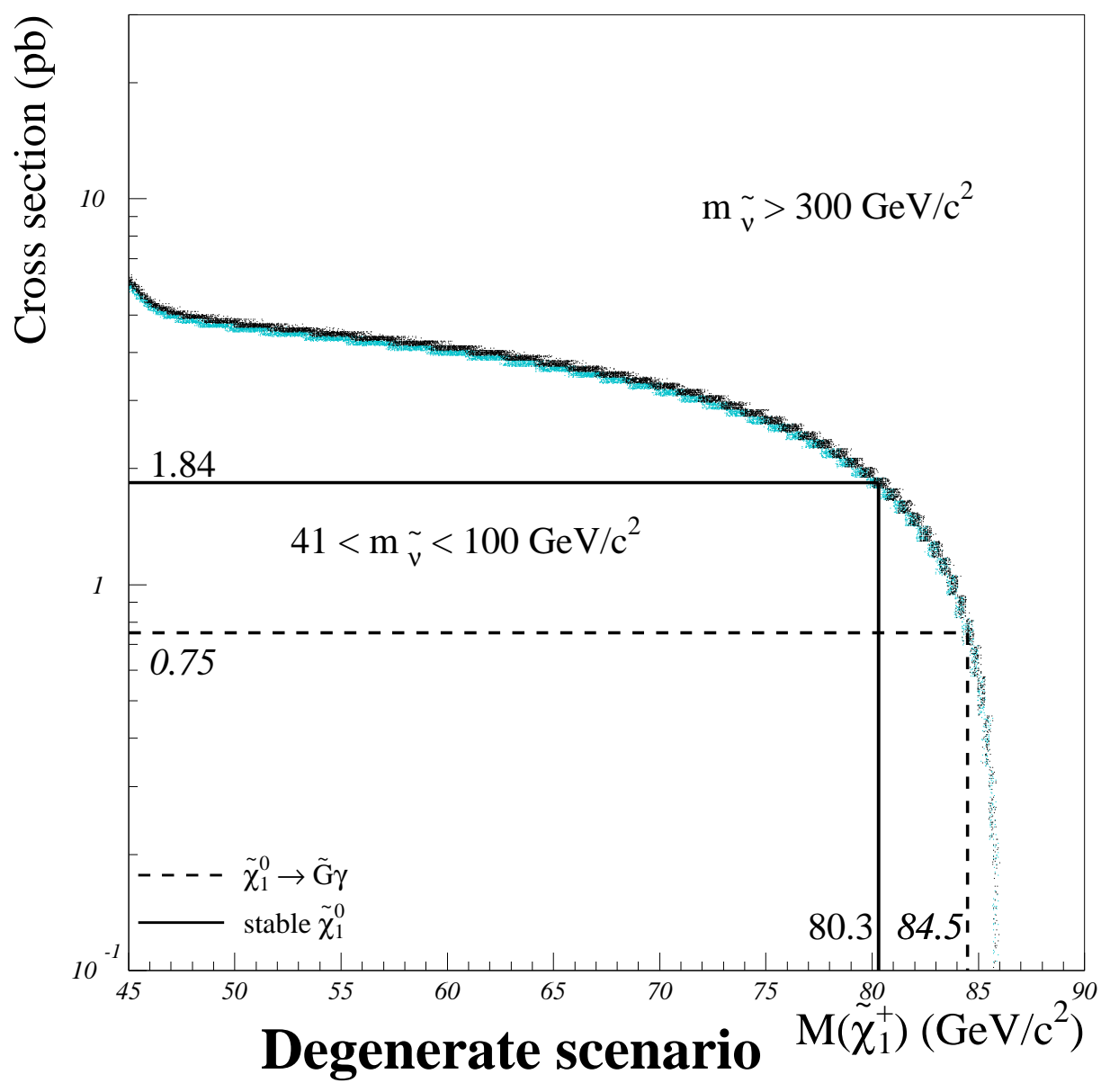

Figure 8: Scatter plot for different MSSM parameters of the expected cross-sections at 172 $\mathrm{GeV}$ versus the chargino mass (dots) in the highly degenerate scenario $\left(\Delta M=5 \mathrm{GeV} / \mathrm{c}^{2}\right.$ for a stable $\tilde{\chi}_{1}^{0}, \Delta M=1 \mathrm{GeV} / c^{2}$ for $\left.\tilde{\chi}_{1}^{0} \rightarrow \tilde{\mathrm{G}} \gamma\right)$. Two different assumptions on the sneutrino mass are made, a light sneutrino $\left(41 \mathrm{GeV} / c^{2}<m_{\tilde{\nu}}<100 \mathrm{GeV} / c^{2}\right)$ and a heavy sneutrino $\left(m_{\tilde{\nu}}>300 \mathrm{GeV} / c^{2}\right)$. The data are practically indistinguishable for these two cases. The corresponding limits on the chargino mass and cross-section are shown. These are independent of the assumed sneutrino mass. 


\section{DELPHI $\tilde{\chi}_{1}^{+} \tilde{\chi}_{1}^{-}$limits at $172 \mathrm{GeV}$}

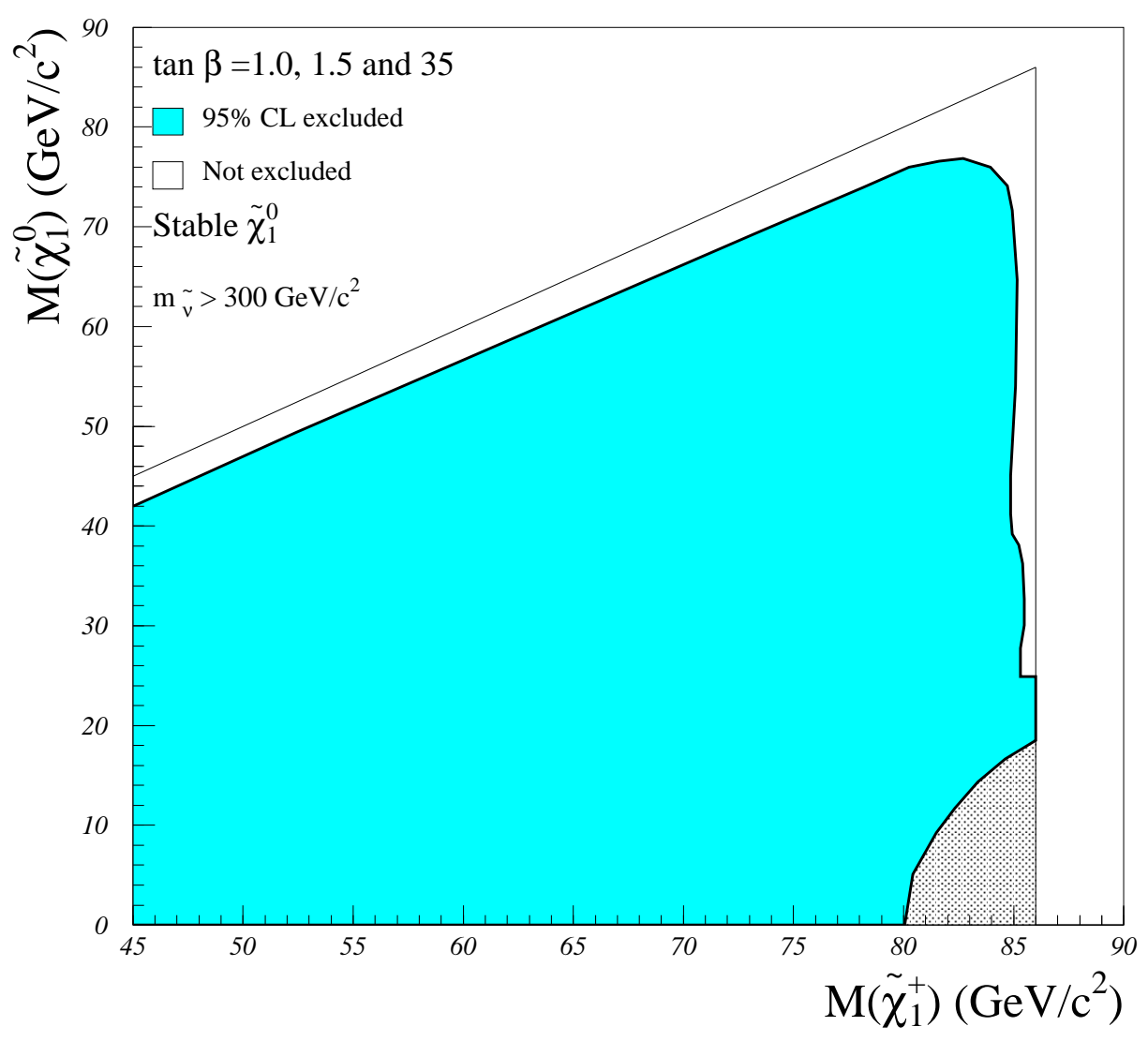

Figure 9: Regions excluded at 95\% confidence level in the plane of the mass of the lightest neutralino versus that of the lightest chargino under the assumption of a heavy sneutrino. The thin lines show the kinematic limits in the production and the decay. The lightly shaded region is not allowed in the MSSM. The limit applies in the case of a stable neutralino. 


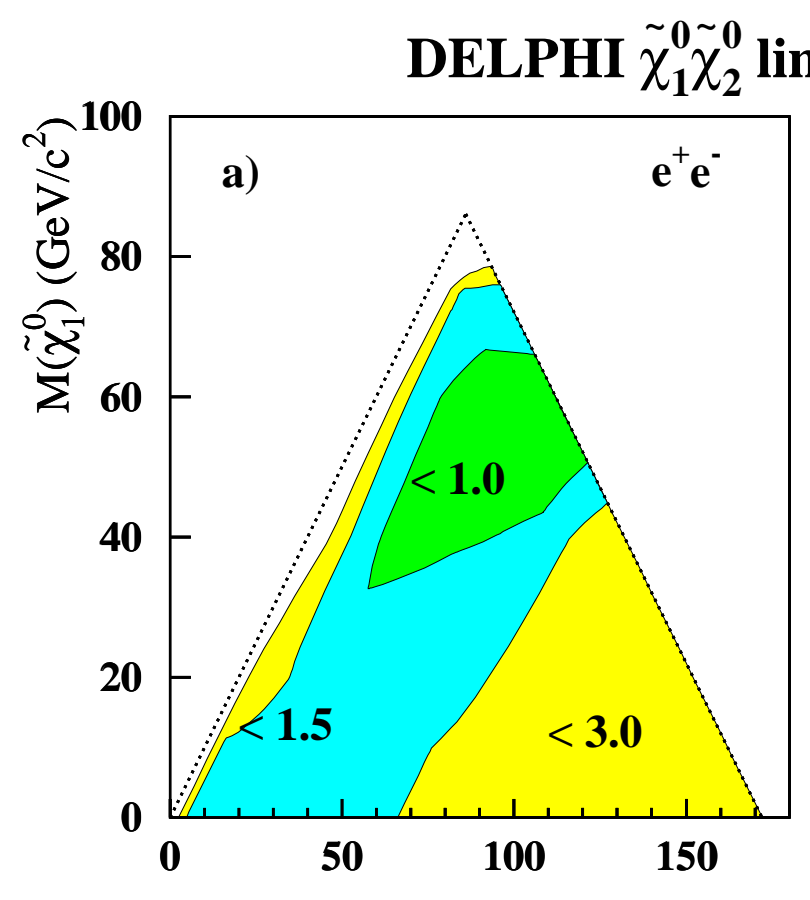

its at $172 \mathrm{GeV}$
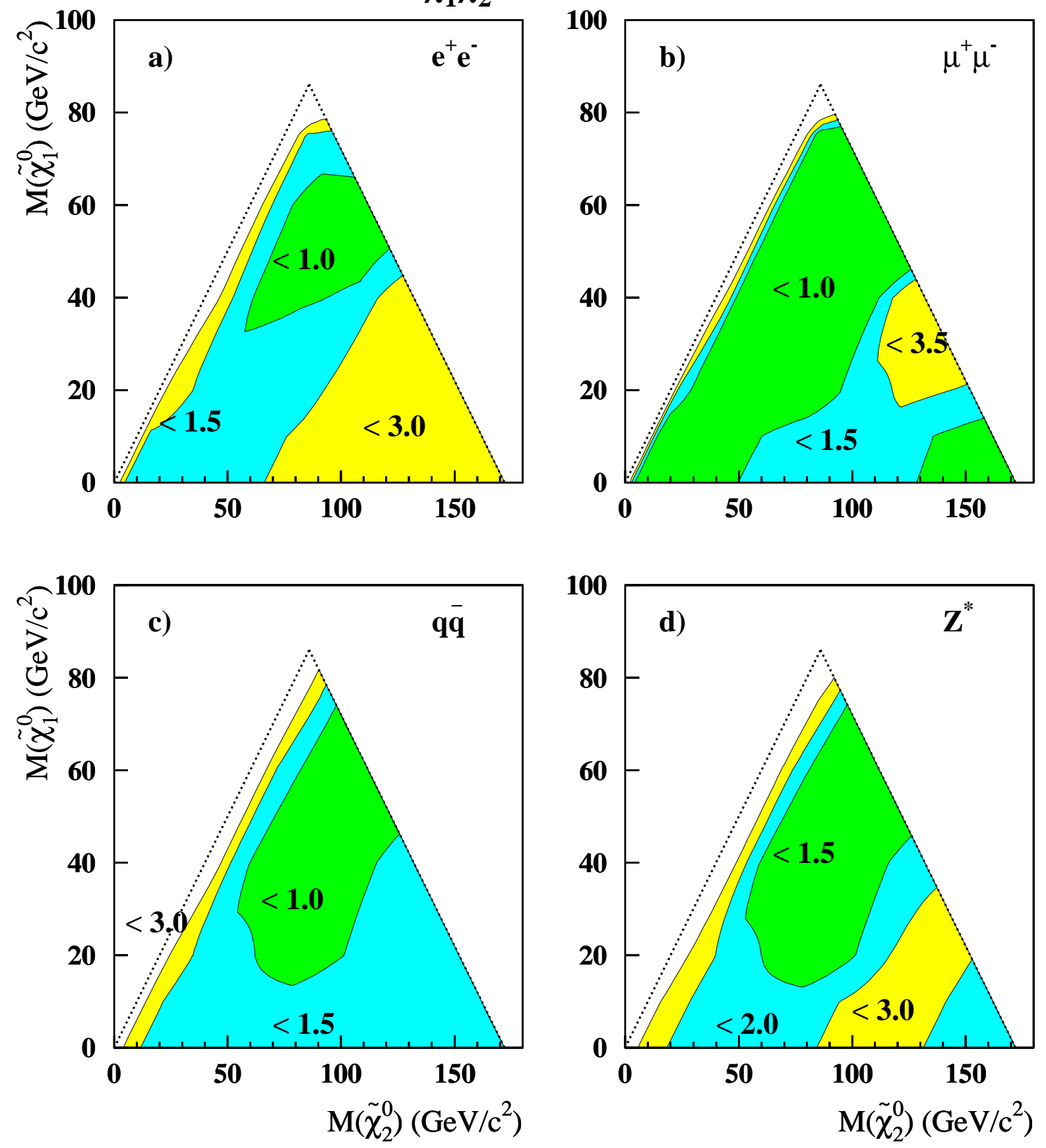

Figure 10: Contour plots of upper limits on the cross-sections at the $95 \%$ confidence level for $\tilde{\chi}_{1}^{0} \tilde{\chi}_{2}^{0}$ production at $\sqrt{s}=172 \mathrm{GeV}$. In each plot, the different shades correspond to regions where the cross-section limit in picobarns is below the indicated number. For figures a), b), c), $\tilde{\chi}_{2}^{0}$ decays into $\tilde{\chi}_{1}^{0}$ and a) $\left.\mathrm{e}^{+} \mathrm{e}^{-}, \mathrm{b}\right) \mu^{+} \mu^{-}$, and c) $\mathrm{q} \bar{q}$, were assumed to dominate. In $\mathrm{d}$ ), the $\tilde{\chi}_{2}^{0}$ was assumed to decay into $\tilde{\chi}_{1}^{0} \mathrm{f} \bar{f}$ with the same branching ratios into different fermion flavours as the $\mathrm{Z}$. The dotted lines indicate the kinematic limit and the defining relation $M_{\tilde{\chi}_{2}^{0}}>M_{\tilde{\chi}_{1}^{0}}$. 


\section{DELPHI MSSM limits}
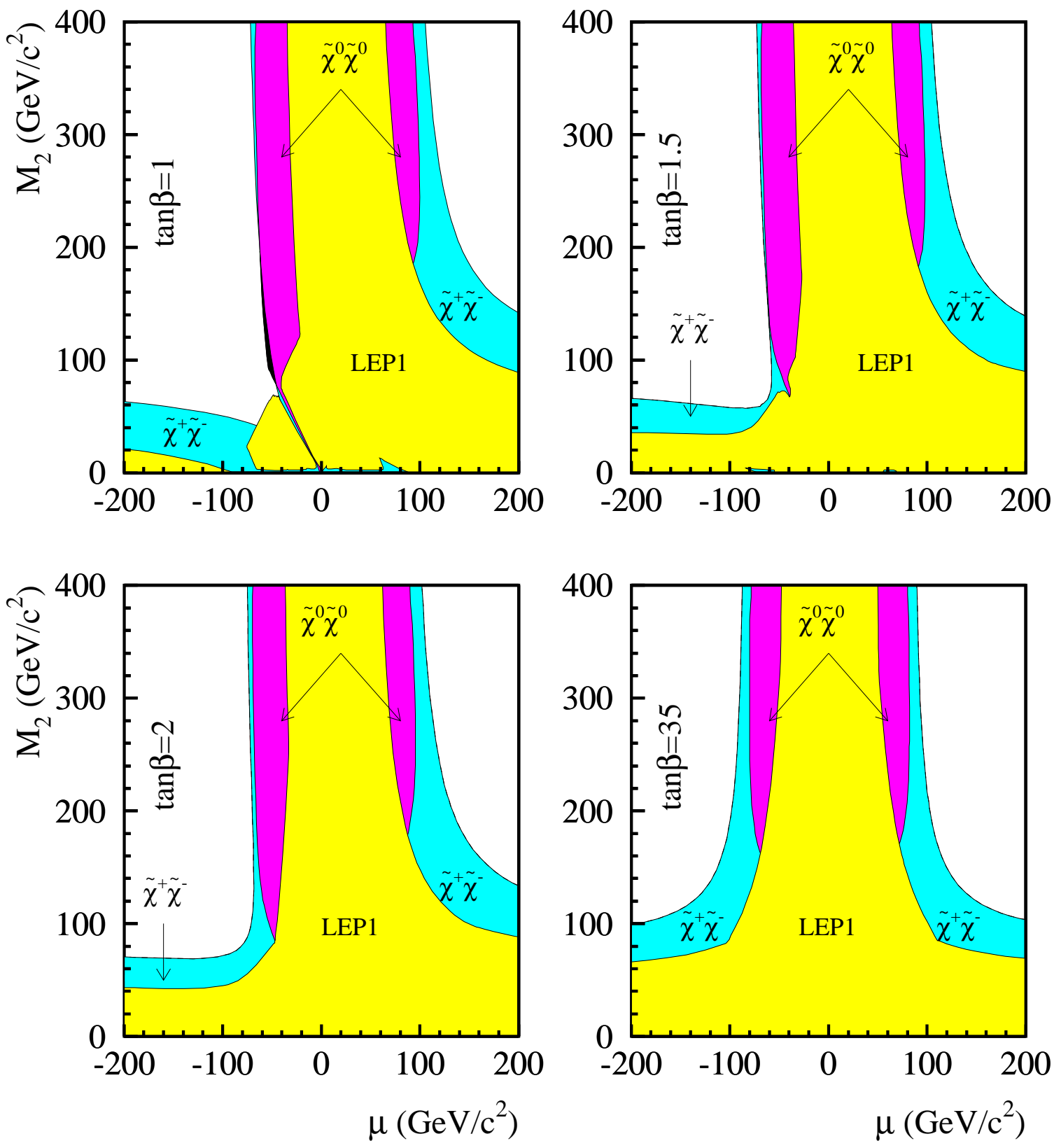

Figure 11: Regions in the $\left(\mu, M_{2}\right)$ plane excluded at $95 \%$ confidence level for different values of $\tan \beta$, assuming $m_{0}=1 \mathrm{TeV} / c^{2}$. The lightly shaded areas are those excluded by lower energy LEP1 results [26]. The intermediate shading shows regions excluded by the chargino search at $161 \mathrm{GeV}$ and $172 \mathrm{GeV}$. The darkly shaded areas show the regions excluded by the neutralino search at these energies. With the exception of a narrow strip at negative $\mu$ for $\tan \beta=1$, indicated in black, the regions excluded by the neutralino results are also excluded by the chargino search. 


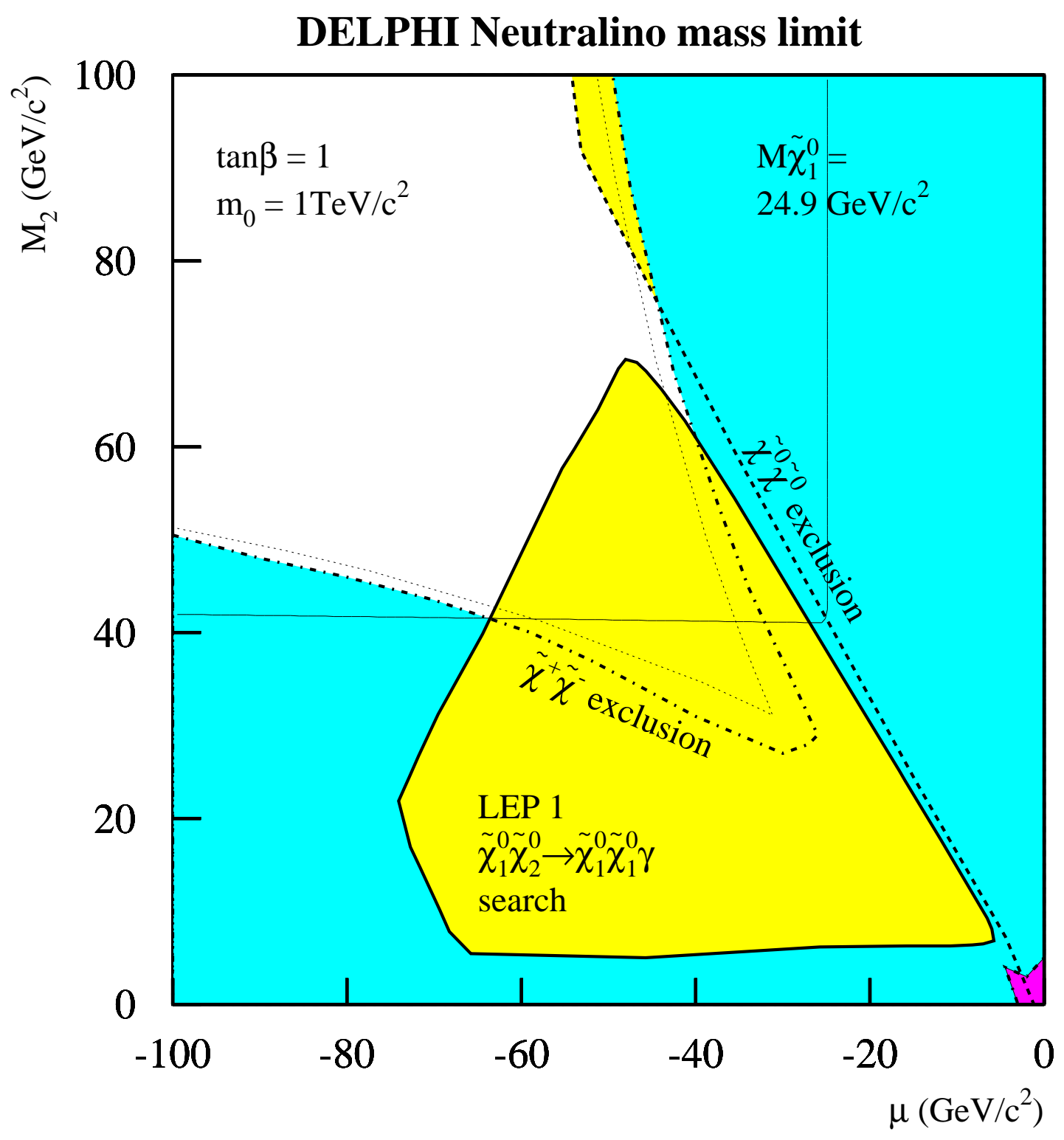

Figure 12: Excluded regions in the part of the $\left(\mu, M_{2}\right)$ plane relevant for the lower limit on the mass of the lightest neutralino. The thick solid line shows the region excluded by the single-photon search at LEP1 [22], the thick dashed-dotted line indicates the exclusion from the chargino searches at $161 \mathrm{GeV}-172 \mathrm{GeV}$. The thick dashed line shows the exclusion from neutralino searches at the same energies. The dark region in the lower right corner is excluded by the limit on anomalous $\mathrm{W}^{+} \mathrm{W}^{-}$production at $172 \mathrm{GeV}$. The thin lines show the $M_{\tilde{\chi}_{1}^{0}}=24.9 \mathrm{GeV} / c^{2}$ contour (solid) and the kinematic limit for $\tilde{\chi}_{1}^{+} \tilde{\chi}_{1}^{-}$ production (dotted). 


\section{DELPHI Neutralino mass limit}

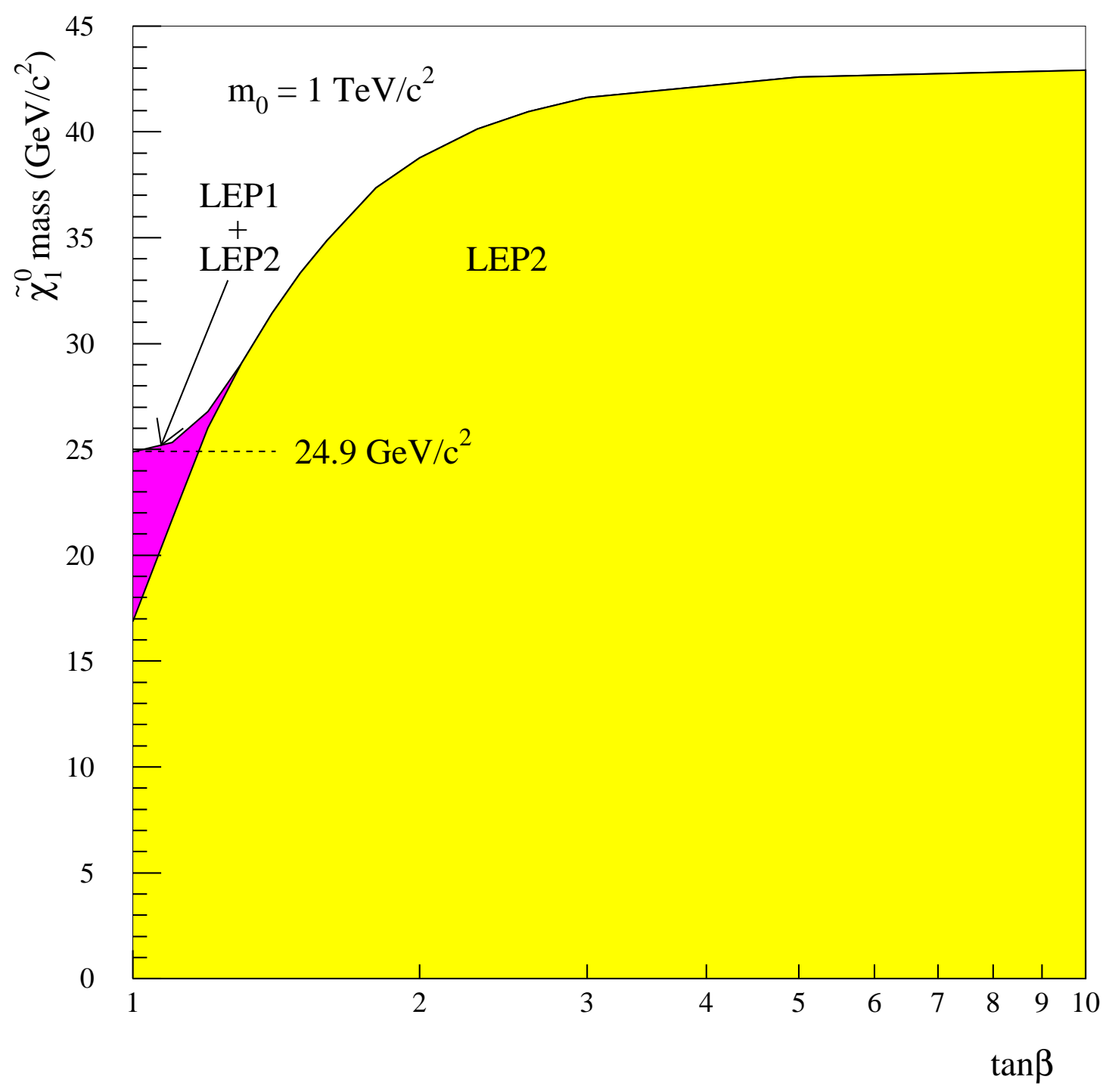

Figure 13: Lower limit at 95\% confidence level on the mass of the lightest neutralino, $\tilde{\chi}_{1}^{0}$, as a function of $\tan \beta$ assuming a stable $\tilde{\chi}_{1}^{0}$ and $m_{0}=1 \mathrm{TeV} / \mathrm{c}^{2}$. The lightly shaded region is excluded by the searches at $\sqrt{s}=161 \mathrm{GeV}$ and $172 \mathrm{GeV}$, while the dark region at low $\tan \beta$ is excluded by the limit on $\tilde{\chi}_{1}^{0} \tilde{\chi}_{2}^{0}$ production at the $Z$ resonance derived from the single-photon search. 


\section{DELPHI $\tilde{\chi}_{1}^{+} \tilde{\chi}_{1}^{-}$efficiencies $(172 \mathrm{GeV})$}
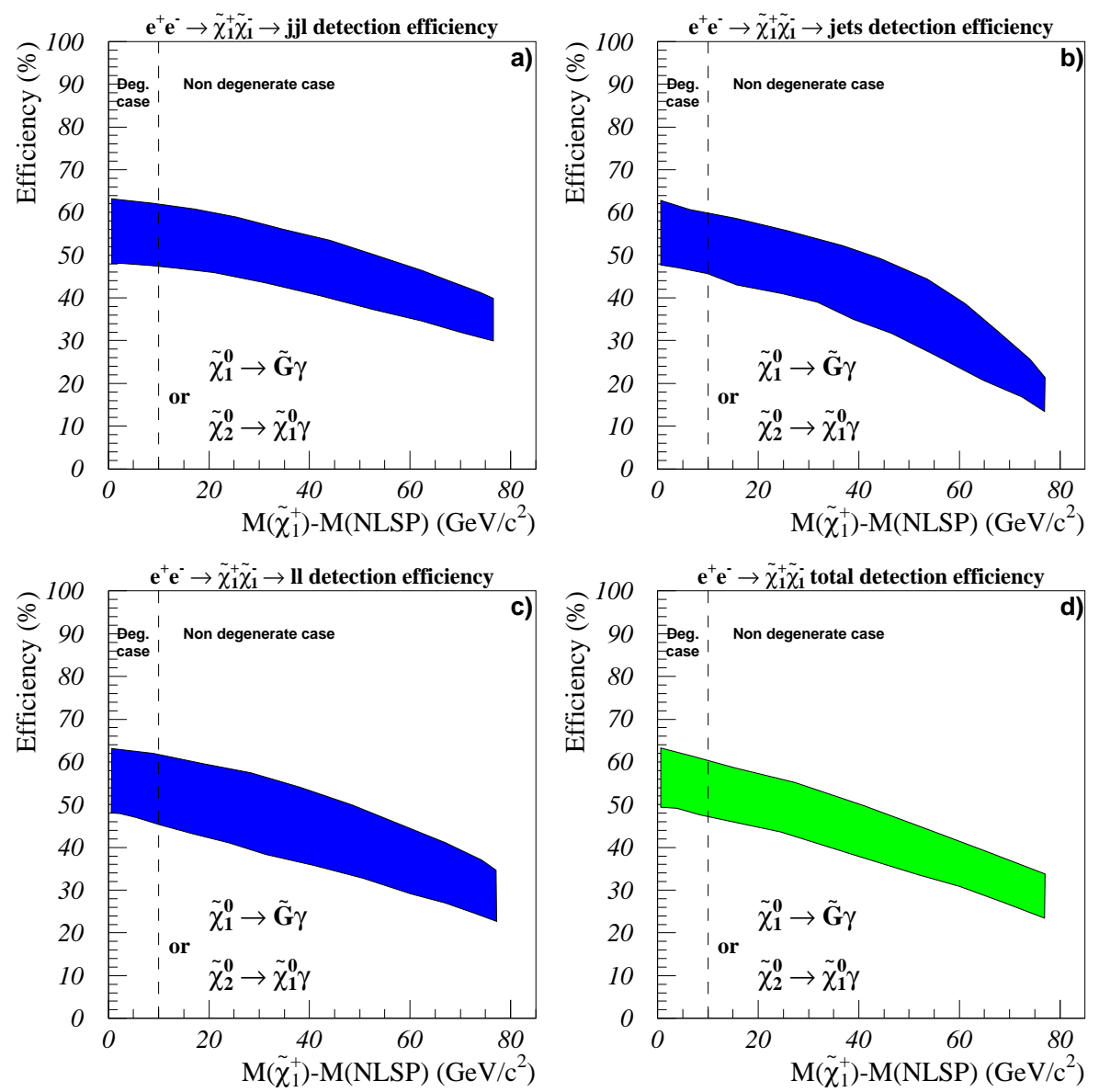

Figure 14: Chargino detection efficiencies in the case of radiative cascade decays via $\tilde{\chi}_{1}^{0}$ or $\tilde{\chi}_{2}^{0}$ into the LSP ( $\tilde{\mathrm{G}}$ or $\tilde{\chi}_{1}^{0}$ ). Details are given in sections 4.4 and 6.1. These efficiencies apply for a) jje, b) jets, c) $\ell \ell$ events with additional photons. d) Overall efficiency. The bands correspond to the statistical error in the simulation of about $2 \%$ absolute, combined with the effect of generating points with different chargino masses. 


\section{DELPHI acoplanar photons}

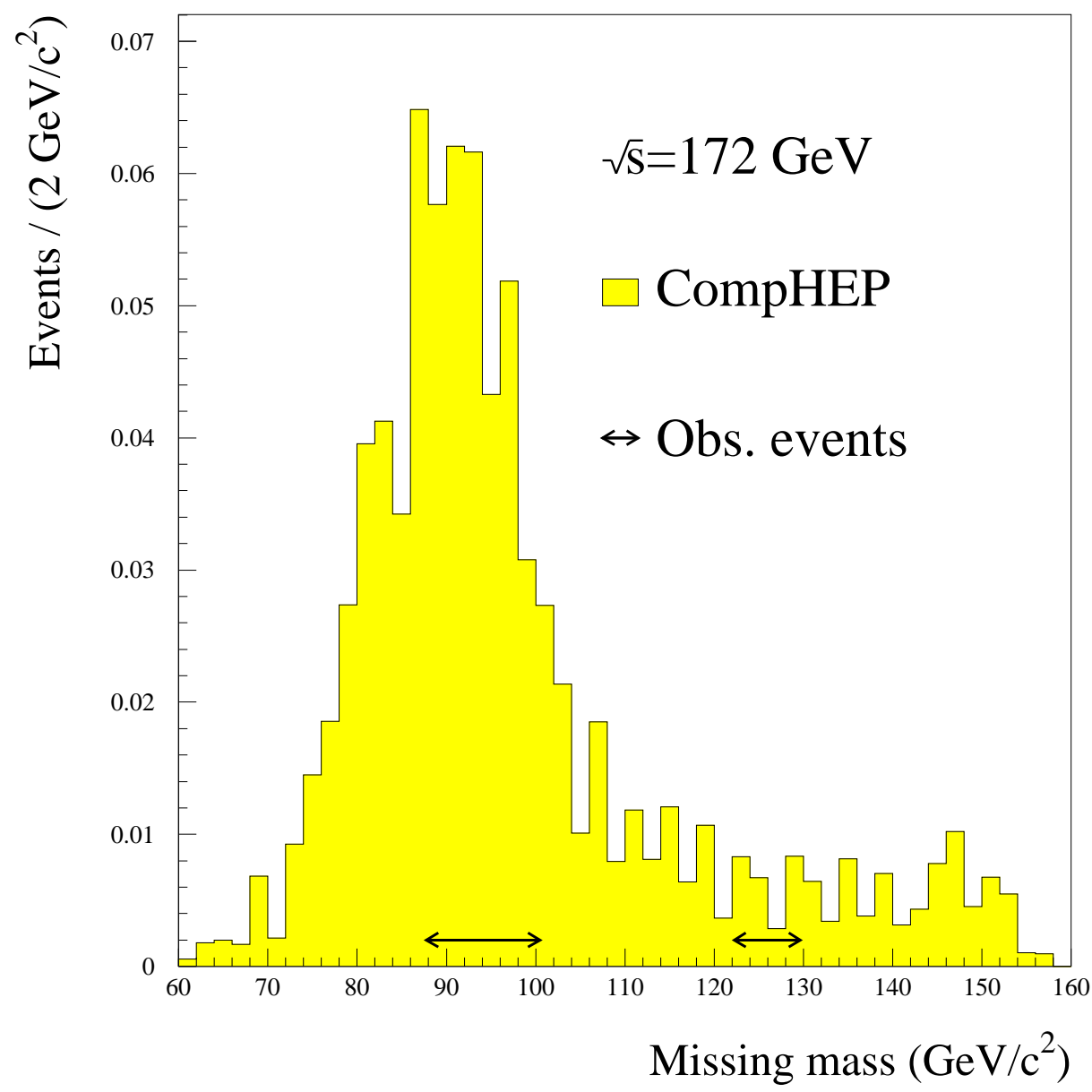

Figure 15: The expected distribution of recoil mass from $\gamma \gamma(\gamma) \nu \bar{\nu}$ final states as calculated using the CompHEP generator including full detector simulation. Also shown are the observed values with errors for the two selected events. 


\section{Acoplanar Photons}

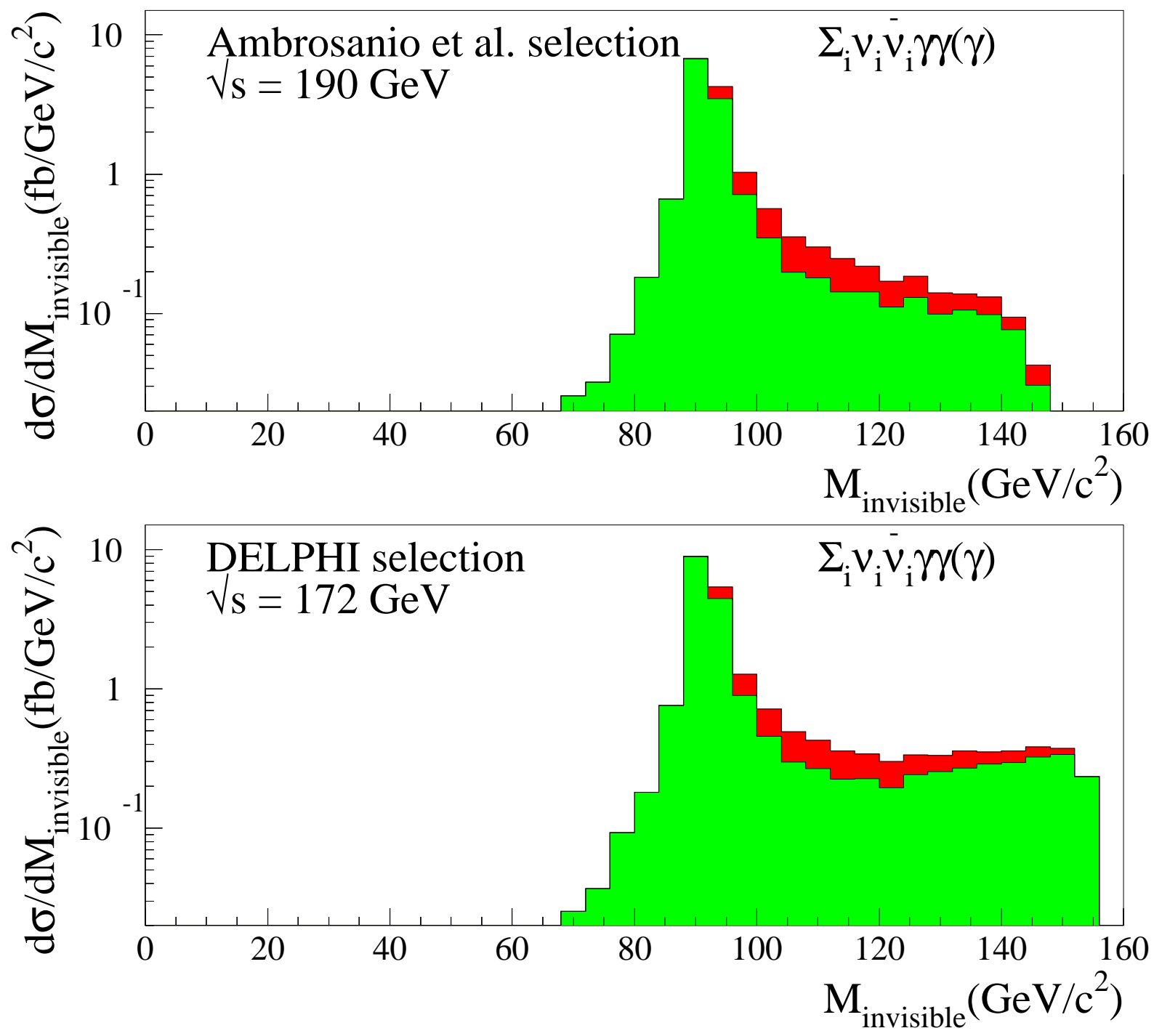

Figure 16: The expected distribution of recoil mass at generator level in $\nu \bar{\nu} \gamma \gamma(\gamma)$ events as calculated using CompHEP. The lightly shaded histogram shows the distribution obtained without including the possibility of a photon from initial state radiation escaping detection at low polar angles. The dark shading shows the increase in cross-section when such radiation is taken into account. The top figure shows the distribution at $\sqrt{s}=190 \mathrm{GeV}$ as obtained using the selection of Ref. [8], the bottom figure shows the corresponding distribution for the present analysis at $\sqrt{s}=172 \mathrm{GeV}$. 

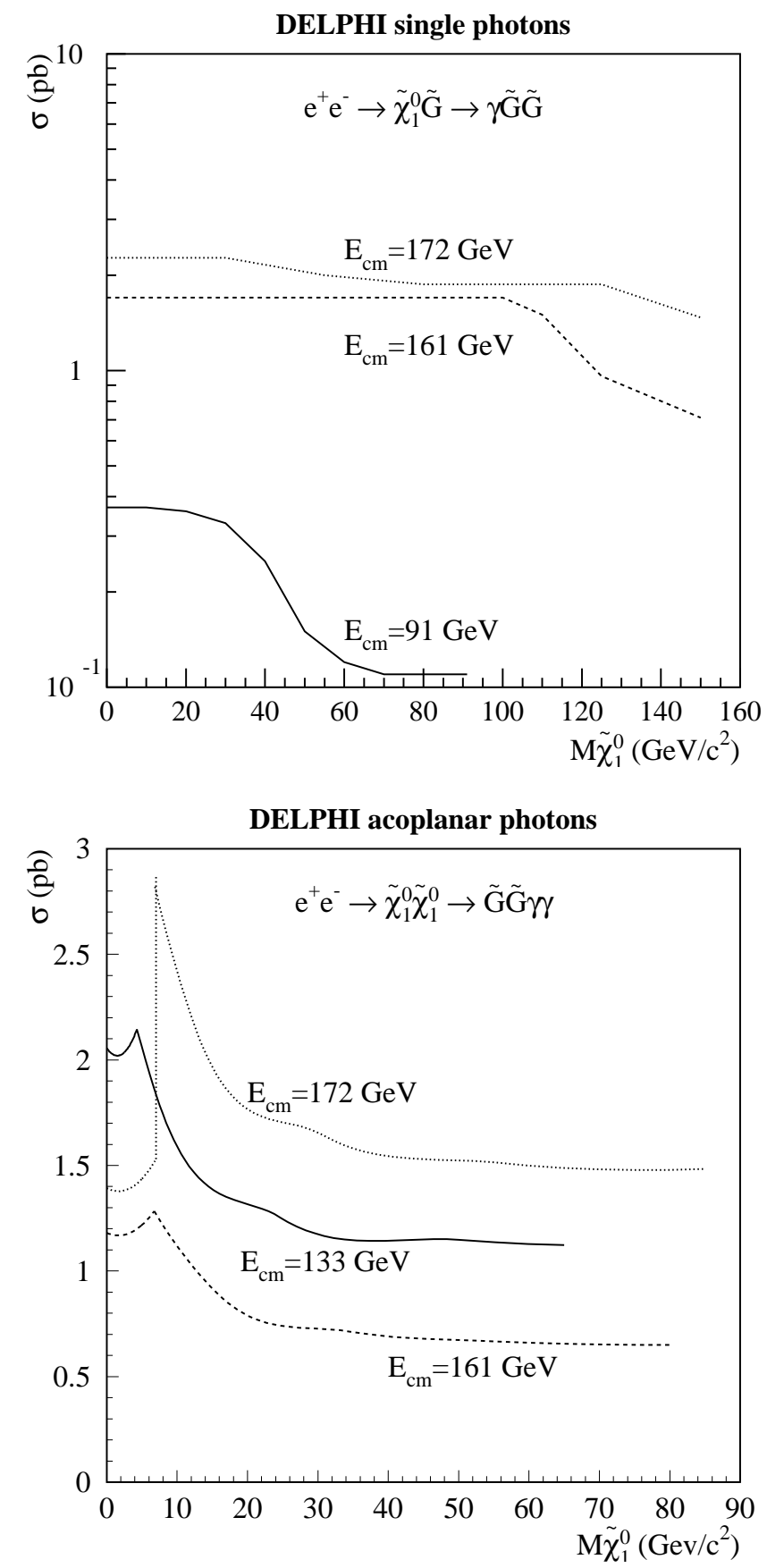

Figure 17: Upper limits at $95 \%$ confidence level on the cross-section for $\tilde{\chi}_{1}^{0} \tilde{G}$ (top) and $\tilde{\chi}_{1}^{0} \tilde{\chi}_{1}^{0}$ (bottom) production at different collision energies as functions of the mass of the lightest neutralino, assuming the decay $\tilde{\chi}_{1}^{0} \rightarrow \tilde{\mathrm{G}} \gamma$. The limits on $\tilde{\chi}_{1}^{0} \tilde{\mathrm{G}}$ production were derived from the analysis of single-photon events and are valid only in case of prompt $\tilde{\chi}_{1}^{0} \rightarrow \tilde{\mathrm{G}} \gamma$ decay, while the limits on $\tilde{\chi}_{1}^{0} \tilde{\chi}_{1}^{0}$ from two photon final states apply for $\tilde{\chi}_{1}^{0}$ decay distances up to about $1 \mathrm{~m}$. The discontinuity in the limit for $172 \mathrm{GeV}$ arises because of the events selected in the high $M_{\tilde{\chi}_{1}^{0}}$ analysis at this energy. 